\title{
Direkte und deliberative Demokratien der Schweiz: Vom Volk, das nicht nur mitredet, sondern entscheidet - und dennoch nicht immer das letzte Wort haben sollte
}

\section{Eva Maria Belser"}

\section{Einleitung}

Die halbdirekte Demokratie der Schweiz ist in mancherlei Hinsicht einzigartig. Schweizerinnen und Schweizer wählen nicht nur periodisch ihre Vertreterinnen und Vertreter im eidgenössischen, in den kantonalen und kommunalen Parlamenten, ihre kantonalen Regierungsmitglieder und teilweise auch ihre Richterinnen und Richter, sie stimmen auch regelmässig über Sachfragen ab. Durchschnittlich drei bis vier Mal im Jahr werden sie an die Urne gerufen, um eidgenössische, kantonale und kommunale Vorlagen gutzuheissen oder abzulehnen. Zwar spielen auch in der Schweiz die repräsentativen Formen der Demokratie eine entscheidende Rolle, doch bemüht sich wohl kaum ein Staat so sehr wie die Schweizerische Eidgenossenschaft darum, eine möglichst weitgehende Übereinstimmung von Herrschern und Beherrschten aufrechtzuerhalten.

Wegen der zahlreichen Vorschlags- und Vetomöglichkeiten der halbdirekten Demokratie war die Schweiz zunächst davon ausgegangen, von den Diskussionen der letzten Jahrzehnte um eine Stärkung der demokratischen Partizipation und um die Einführung deliberativer Formen nicht berührt zu sein. Angesichts verschiedener als problematisch empfundener Volksentscheide schien nicht mehr, sondern weniger Mitbestimmung das Thema der Stunde zu sein. Dabei wurde allerdings übersehen, dass neuere demokratietheoretische Strömungen weniger auf ein quantitatives Mehr an Volksentscheiden zielen, als vielmehr eine Ausweitung der Demokratie auf gesellschaftliche und wirtschaftliche Bereiche oder ihre Vertiefung durch eine möglichst frühe, breite und intensive Beteiligung des Volks anstreben. Diese Bemühungen, die Qualität der politischen Mitwirkung zu verbessern, sind auch für die halbdirekten Demokratien der Schweiz von

* Ich danke meiner Mitarbeiterin Ilona Kessler sowie meinem Mitarbeiter Simon Mazidi herzlich für ihre wertvolle Unterstützung bei der Vorbereitung und der redaktionellen Überarbeitung dieses Texts. 
Bedeutung und stehen Auseinandersetzungen um rechtliche Schranken der Mitbestimmung nicht entgegen.

Der vorliegende Beitrag setzt sich mit den unterschiedlichen direkten und deliberativen Formen der Demokratien der Schweiz auseinander. Als direkte Formen gelten dabei jene Mitwirkungsrechte, die es den Stimmberechtigten (und nur diesen) ermöglichen, durch Mehrheitsentscheid verbindliche Sachentscheide selbst zu treffen. Als deliberative Formen werden dagegen jene Partizipationsformen bezeichnet, die die Gestaltungs- und Einflussmöglichkeiten der Bevölkerung erweitern, ohne dieser zusätzliche Entscheidbefugnisse einzuräumen. Deliberative Demokratie zielt darauf, Foren zu schaffen, die es der breiten Bevölkerung oder von geplanten Entscheiden besonders betroffenen Menschen (die nicht notwendigerweise Stimmberechtigte sein müssen) ermöglichen, sich in staatliche Entscheidungsprozesse einzubringen und einen Einfluss auf diese auszuüben.

Bei der Frage nach der demokratischen Mitbestimmung in der Schweiz gilt es der Tatsache Rechnung zu tragen, dass es die Schweizerische Demokratie nicht gibt, sondern aufgrund der Vielfalt der demokratischen Einflussmöglichkeiten auf kantonaler und kommunaler Ebene von einer Mehrzahl Schweizerischer Demokratien auszugehen ist. Ebenfalls darf nicht vergessen werden, dass die Unterscheidung zwischen direkten und deliberativen Formen der Demokratie keine scharfe ist, sondern dass die verschiedenen Formen auf vielfältige Weise miteinander verbunden sind und ineinander übergehen. Auch wenn seit einigen Jahren vor allem auf kantonaler und kommunaler Ebene mit neuen Formen der deliberativen Demokratie experimentiert wird, wäre es doch falsch, neue Formen der Deliberation den klassischen Volksrechten gegenüber zu stellen. Vor allem die Möglichkeit des fakultativen Referendums hat in der Schweiz seit jeher dazu geführt, dass Bürgerinnen und Bürger früh über Gesetzgebungsvorhaben informiert und zu Stellungnahmen eingeladen werden und dass deren Bedenken oft schon deshalb Rechnung getragen wird, um zu verhindern, dass später Referenden ergriffen und Vorhaben in Volksabstimmungen zu Fall gebracht werden. In einem Land mit vielfältigen Interventionsmöglichkeiten können Repräsentantinnen und Repräsentanten nicht lange am Volk vorbeiregieren, sondern müssen, wollen sie nicht durch Initiativ- und Referendumsstürme lahmgelegt werden, seine Befindlichkeiten aufmerksam in Erfahrung bringen und seinen Prioritäten angemessen Rechnung tragen.

Dank der Abstimmungsdemokratie ist die Politikverdrossenheit in der Schweiz weniger stark ausgeprägt als in anderen Staaten. Ging man bis vor kurzem noch davon aus, dass die Stimmbeteiligung von meist deutlich 
unter 50 Prozent der Qualität der Demokratie schade, ${ }^{1}$ so zeigen neue Forschungsergebnisse, dass sich nur 10 Prozent der Stimmberechtigten gänzlich der Mitwirkung enthalten; die übrigen 90 Prozent sind politisch aktiv und nehmen an Abstimmungen teil, wenn sie Interesse am Thema haben. ${ }^{2}$ Auch der Populismus fällt in der Schweiz nicht auf den gleichen Nährboden, wie dies in rein repräsentativen Demokratien der Fall ist. Aufgrund der engen Verschränkung zwischen den politischen Eliten, die unter ständiger Beobachtung stehen und nicht selten durch Volksentscheide desavouiert werden, fällt es weniger leicht, das politische Establishment gegen das Volk auszuspielen. Gleichzeitig machen Parteien, wie etwa die Schweizerische Volkspartei (SVP), die teilweise populistische Haltungen vertritt, immer wieder die Erfahrung, in Wahlen zwar vergleichsweise erfolgreich abzuschneiden, an der Urne aber immer wieder Abstimmungen zu verlieren; sie kann deshalb weniger glaubwürdig als andere populistische Strömungen für sich in Anspruch nehmen, das "wahre«, homogene Volk zu vertreten.

Wegweisend für den Gang der Untersuchung ist aber noch eine andere Frage, ohne die der Blick auf die Schweizerische Demokratie ein unvollständiger wäre: die Frage nach den Schranken der demokratischen Mitwirkung. Noch öfter als die Forderung nach einer Erweiterung oder Vertiefung der demokratischen Mitwirkung wird in der Schweiz der Wunsch geäussert, dem Volk doch auch einmal das Schweigen zu gebieten und jene Formen der Demokratie zu beschränken, die zwar über eine hohe Input-Legitimität verfügen (weil »der Souverän" gesprochen hat), deren Output-Legitimität aber fraglich ist, weil Volksentscheide Einzelne oder Minderheiten benachteiligen oder zu anderen rechtstaatlichen Schwierigkeiten führen, etwa die Einhaltung der völkerrechtlichen Pflichten des Landes erschweren.

Nicht Thema der Abhandlung sind die repräsentativen Formen der Demokratie, die als bekannt vorausgesetzt werden, sowie jene Forderungen der partizipativen Demokratietheorie, die auf die Ausdehnung demokrati-

1 Im Zeitraum zwischen 2011 und 2017 lag die Stimmbeteiligung auf Bundesebene bei durchschnittlich 46,4\%, für die Jahre 1991 bis 2011 lag sie leicht darunter bei 44,1\%; vgl. Bundesamt für Statistik, »Entwicklung der Stimmbeteiligung bei eidgenössischen Volksabstimmungen« (2017) www.bfs.admin.ch/bfs/de/home/statistike $\mathrm{n} /$ politik/abstimmungen/stimmbeteiligung.assetdetail.3602770.html (abgerufen am 4. Januar 2018).

2 Vgl. Andreas Goldberg, Simon Lanz und Pascal Sciarini, "Abstimmungen: Nur wenige gehen immer oder nie an die Urne« (DeFacto, 15. Februar 2016) www.defacto. expert/2016/02/15/selektive-partizipation/ (abgerufen am 4. Januar 2018). 
scher Mitwirkungsrechte auf Arbeit, Familie und andere gesellschaftliche Bereiche zielen. Im Folgenden werden zuerst kurz die Formen der direkten Demokratie in Erinnerung gerufen (2.) und deren Schranken erläutert (3.). In Anschluss daran werden alte und neue Formen deliberativer Demokratie vorgestellt (4.) und die Ergebnisse zusammengefasst (5.).

\section{Formen der direkten Demokratie}

In halbdirekten Demokratien wie jenen der Schweiz gibt es zwar Parlamente und Regierungen, die dazu berufen sind, den Willen des Volks zum Ausdruck zu bringen und in seinem Namen zu handeln, doch können die Stimmberechtigten in bestimmten Fällen oder unter bestimmten Umständen auch selbst Sachentscheide fällen. Die direktdemokratischen Mitwirkungsrechte des Volks erweitern die Einfluss- und Gestaltungsmöglichkeiten der stimmberechtigten Bevölkerung und gelten insofern als besonders demokratisch, als Volksentscheide jede Verfälschung des Volkswillens ausschliessen, die sich aufgrund der Vertretung ergeben können. Misst man die Demokratie an der Übereinstimmung zwischen Herrschern und Beherrschten, schneidet die direkte Demokratie gegenüber allen Formen der Demokratie, die auf mehr oder weniger langen Legitimationsketten beruhen, am besten ab. Ihre Input-Legitimität (Herrschaft durch das Volk) ist zumindest dann sehr hoch, wenn das Volk seinen Entscheid frei und informiert fällt (weniger aber, wenn sein Entscheid durch windige Demagogen, intransparentes Lobbying und skandalhungrige Medien manipuliert wird). Dass das Volk selbst entscheidet, führt jedoch nicht zwingend zu einer erhöhten Output-Legitimität (Herrschaft für das Volk). Politische Sachentscheide, die das Volk fällt, bergen vielmehr die Gefahr, dass eine Mehrheit (oder eine gut organisierte Minderheit) bestimmte Gruppen oder Personen benachteiligt oder deren Rechte beschränkt und werfen die Frage nach den Schranken der Mehrheitsdemokratie auf.

Im Folgenden werden die direktdemokratischen Mechanismen der Schweizer Demokratien nach Vorschlags- und Vetorechten unterschieden. Während Initiativrechte es dem Volk ermöglichen, gestaltend auf politische Entscheide einzuwirken und staatliches Handeln anzustossen, geben ihm Referendumsrechte die Gelegenheit, staatliches Handeln durch Volksentscheide zu blockieren und gegenüber Entscheiden seiner Vertreterinnen und Vertreter ein Veto einzulegen. Da es die Vorschlagsrechte dem Volk erlauben, vom Parlament nicht berücksichtigte oder vernachlässigte Themen auf die Agenda zu setzen und Gesetzgebungsakte auch gegen den Willen von Parlament und Regierung zu erzeugen, nehmen die Vor- 
schlagsrechte eine Ventilfunktion wahr. Sie erlauben es politisch aktiven Gruppierungen, die Berücksichtigung ihrer Anliegen zu erzwingen, ohne auf ausserrechtliche Instrumente zurückgreifen zu müssen (positiver Gesetzgeber). Während die Vorschlagsrechte beschleunigen (oder lenken), bremsen (oder stoppen) die Vetorechte und nehmen eine Rotlichtfunktion ein. Sie erlauben es dem Volk einzugreifen, wenn es mit der Art und Weise, wie seine Repräsentantinnen und Repräsentanten die Vertretung wahrnehmen, nicht einverstanden ist (negativer Gesetzgeber).

\subsection{Direkt-demokratische Vorschlagsrechte: Die Ventilfunktion}

Volksinitiativen geben dem Volk (bzw. Bevölkerungsgruppen) die Möglichkeit, eine gesetzliche Änderung vorzuschlagen oder eine solche anzustossen. Sie sind auf allen Ebenen des Schweizerischen Bundesstaats vorgesehen und lösen die Durchführung verbindlicher Volksentscheide aus.

\subsubsection{Die Volksinitiativen auf Bundesebene}

Auf der Ebene des Bundes ist als Vorschlagsrecht die Volksinitiative auf Total- und auf Teilrevision der Bundesverfassung vorgesehen. ${ }^{3}$ Regen 100000 stimmberechtigte Schweizerinnen und Schweizer eine Totalrevision der Bundesverfassung an, so findet zwingend eine Volksabstimmung über die Frage statt, ob die Grundlagen des Staates umfassend neu kodifiziert werden sollen. Die Initianten haben sich in diesem Fall auf eine allgemeine Anregung zu beschränken und müssen die Ausarbeitung der neuen Verfassung der Bundesversammlung überlassen. Seit der Gründung des Bundesstaats im Jahre 1848 wurde der Vorschlag einer Totalrevision viermal, zuletzt im Jahre 2003, durch Volksinitiative vor das Stimmvolk gebracht, jedoch jedes Mal von Volk und Ständen abgelehnt. ${ }^{4}$

In der Praxis bedeutend ist denn auch nicht die Volksinitiative auf Total- , sondern jene auf Teilrevision der Bundesverfassung, die im Jahre

3 Art. 138 f. sowie Art. 192 ff. Bundesverfassung der Schweizerischen Eidgenossenschaft vom 18. April 1999 (SR 101, BV); Ulrich Häfelin, Walter Haller, Helen Keller und Daniela Thurnherr, Schweizerisches Bundesstaatsrecht (Schulthess, 2016), 414.

4 Astrid Epiney und Stefan Diezig, »Kommentar zu Art. 138 BV«, in Bernhard Waldmann, Eva Maria Belser und Astrid Epiney (Hrsg.), Basler Kommentar - Bundesverfassung (Helbling Lichtenhahn, 2015), N 6. 
1891 eingeführt worden ist und seither zu einem wichtigen Charakteristikum der Schweizerischen Demokratie geworden ist. Sie erlaubt es Stimmberechtigen, in der Form einer allgemeinen Anregung oder eines ausgearbeiteten Entwurfs zu verlangen, dass eine oder mehrere Bestimmungen der Verfassung geändert, aufgehoben oder neu eingefügt werden. Gelingt es dem Initiativkomitee (einer Partei, einem Verein, einer Interessengruppe oder einer ad hoc zusammengesetzten Personengruppe) innerhalb von 18 Monaten die Unterschriften von 100000 Stimmberechtigten für ihr Anliegen zu sammeln, so ist die Initiative zustande gekommen. ${ }^{5}$

In der Praxis wählen Initiativkomitees auf Bundesebene (im Unterschied zu jenen auf kantonaler Ebene) ausschliesslich die Form des ausgearbeiteten Entwurfs. Erfüllt dieser die Gültigkeitserfordernisse, muss er zwingend und unverändert Volk und Ständen zur Abstimmung vorgelegt werden und tritt mit seiner Gutheissung unmittelbar in Kraft. ${ }^{6}$ Der Bundesversammlung bleibt einzig die Möglichkeit, die Volksinitiative Volk und Ständen zur Ablehnung zu empfehlen oder der Initiative einen (gemässigteren) Gegenentwurf gegenüberzustellen, der gleichzeitig zur Abstimmung kommt (direkter Gegenvorschlag). ${ }^{7}$ Weil die Bundesverfassung keine anderen Vorschlagsrechte, namentlich keine Gesetzesinitiative kennt, kann es vorkommen, dass Initiativkomitees gesetzgeberische Anliegen in der Form einer Volksinitiative auf Teilrevision der Bundesverfassung vorlegen (z.B. eine Verbesserung der Raumplanung oder eine Verschärfung des Ausländer- oder Strafrechts fordern). In diesem Fall kann die Bundesversammlung eine Gesetzesänderung im Sinne der Initiantinnen und Initianten beschließen (indirekter Gegenvorschlag) und darauf hoffen, dass diese die Initiative zurückziehen oder dass die Initiative vor Volk und Ständen keine Mehrheit findet, weil das Anliegen bereits als (weitgehend) erfüllt gilt.

Seit der Einführung der eidgenössischen Volksinitiative auf Teilrevision der Bundesverfassung wurden für insgesamt 455 Volksinitiativen Unterschriften gesammelt. ${ }^{8}$ Von diesen Initiativen kamen 328 gültig zustande, während 116 im Unterschriftenstadium scheiterten. Nur vier Volksinitia-

5 Art. 139 BV; Häfelin et al., Schweizerisches Bundesstaatsrecht (Fn. 4), 544.

6 Art. 195 BV; Art. 99 Bundesgesetz über die Bundesversammlung vom 13. Dezember 2002 (SR 171.10, ParlG).

7 Art. 139b BV; Art. 101 f. ParlG.

8 Vgl. Bundeskanzlei BK, »Übersicht in Zahlen« (2017) www.admin.ch/ch/d/pore/vi/ vis_2_2_5_9.html (abgerufen am 4. Januar 2018). 
tiven wurden von der Bundesversammlung für ungültig erklärt. ${ }^{9}$ Von den 209 Volksinitiativen auf Teilrevision der Bundesverfassung, die zur Abstimmung gelangten, wurde die große Mehrzahl abgelehnt. Nur 22 fanden das qualifizierte Mehr, die meisten davon seit den 1980er Jahren. ${ }^{10}$ Gegenwärtig befinden sich 26 Volksinitiativen in Vorbereitung, ${ }^{11}$ was die zunehmende Beliebtheit des Initiativrechts unterstreicht. Auch zahlreiche abgelehnte Initiativen, namentlich solche, die von einem erheblichen Teil der Bevölkerung unterstützt wurden, haben oft deutliche Spuren in Politik und Recht hinterlassen und ihre Funktion, ein Thema auf die politische Agenda zu setzen, erfüllt.

Das Initiativrecht wird oft als Ventil bezeichnet, weil es der Bevölkerung erlaubt, Themen, die als wichtig, aber vernachlässigt erachtet werden, aufzugreifen und ihnen die Aufmerksamkeit von Politik, Medien und weiterer Öffentlichkeit zu verschaffen. Wer sich über eine Entwicklung oder ihr Fehlen empört oder sich mit einem Thema politisch profilieren will, kann zum Unterschriftenbogen greifen und muss nicht auf die nächsten Wahlen warten oder auf der Straße oder in den sozialen Medien politischen Druck erzeugen. Wer findet, dass die Vertreterinnen und Vertreter am Volk vorbeipolitisieren, kann es auf eine Probe aufs Exempel ankommen lassen; hat die Initiative Erfolg, wird die Bundesverfassung entsprechend angepasst, scheitert sie aber, können ihre Verfechterinnen und Verfechter nicht mehr ohne weiteres für sich in Anspruch nehmen, das Volk gegen korrumpierte Vertreterinnen und Vertreter zu verteidigen.

9 Die Durchsetzungsinitiative wurde für teilweise ungültig erklärt; Bundeskanzlei BK, »Ungültig erklärte Volksinitiativen « (2017) www.bk.admin.ch/ch/d/pore/vi/vi s_2_2_5_6.html (abgerufen am 4. Januar 2018); Pierre Tschannen, Staatsrecht der Schweizerischen Eidgenossenschaft (Stämpfli Verlag, 2016), 572.

1015 der 22 Volksinitiativen wurden seit 1982 angenommen und 10 alleine seit der Jahrtausendwende; vgl. Bundeskanzlei BK, »Von Volk und Ständen angenommene Volksinitiativen « (2017) www.bk.admin.ch/ch/d/pore/vi/vis_2_2_5_8.html (abgerufen am 4. Januar 2018).

11 Zwei Volksinitiativen sind abstimmungsreif, acht vor dem Parlament und fünf vor dem Bundesrat hängig, eine Initiative wird von der Staatskanzlei vorgeprüft (Auszählung) und zehn befinden sich im Sammelstadium; vgl. Bundeskanzlei BK, »Hängige Volksinitiativen« (2017) www.admin.ch/ch/d/pore/vi/vis_1_3_1_1. html (abgerufen am 4. Januar 2018). 


\subsubsection{Die Volksinitiativen auf kantonaler und kommunaler Ebene}

Die 26 Kantone der Schweiz genießen eine weitgehende Organisationsautonomie und bestimmen im Wesentlichen selbst über die Ausgestaltung ihrer Demokratie. Die Bundesverfassung verlangt von den Kantonen lediglich, dass sie sich eine demokratische Verfassung geben; diese bedarf der Zustimmung des Volks und muss revidiert werden, wenn die Mehrheit der Stimmberechtigten es verlangt. ${ }^{12}$ Während die bundesstaatliche Homogenitätsklausel damit nur vorschreibt, dass die Verfassung einem obligatorischen Referendum unterliegt und Volksinitiativen auf Verfassungsänderungen zulässig sein müssen, gehen sämtliche Kantone weit über diese Mindestanforderungen hinaus und kennen eine Vielzahl unterschiedlich ausgestalteter direktdemokratischer Vorschlagsrechte. Andreas Auer bezeichnet die Schweizer Kantone denn auch als »die weltweit wohl demokratischsten Körperschaften ${ }^{13}$. Sie blicken meist auf eine jahrhundertelange Tradition direktdemokratischer (zumindest androkratischer) Regierungsformen zurück, die auf genossenschaftliche Traditionen und dem Zunftwesen beruhen und meist viel älter sind als die eidgenössischen Volksrechte.

Sämtliche Kantone sehen neben der Volksinitiative auf Total- oder Teilrevision der Verfassung auch die Gesetzesinitiative vor, mit der Stimmberechtigte den Erlass, die Änderung oder Aufhebung von Gesetzen verlangen können. ${ }^{14}$ Wie auf Bundesebene können Volksinitiativen auf einem ausgearbeiteten Vorschlag oder einer allgemeinen Anregung beruhen, die das Parlament zur Ausarbeitung einer Vorlage verpflichtet. In allen Kantonen können die Parlamente den Volksinitiativen eigene Vorschläge gegenüberstellen und diese gleichzeitig mit der Initiative zur Abstimmung bringen. ${ }^{15}$ In einigen Kantonen haben die Stimmbürgerinnen und Stimmbürger außerdem die Möglichkeit, eine Volksinitiative über Verordnungen, bestimmte Beschlüsse, Staatsverträge oder interkantonale Konkordate zu ergreifen. Sie können teilweise auch direktdemokratisch verlangen, dass der Kanton eine Standesinitiative einreicht und sich mit einem Anliegen an die Bundesversammlung wendet, ${ }^{16}$ oder mit Hilfe einer Volksmotion

12 Art. 51 Abs. 1 BV; Eva Maria Belser und Nina Massüger, »Kommentar zu Art. 51 $\mathrm{BV} \lll$, in Waldmann, Belser und Epiney (Hrsg.), Basler Kommentar (Fn. 5), N 18 ff. und $37 \mathrm{ff}$.

13 Andreas Auer, Staatsrecht der Schweizerischen Kantone (Stämpfli Verlag, 2016), 413.

14 Zum Ganzen ebd., 426 ff.

15 Ebd., $436 \mathrm{ff}$.

16 Vgl. Art. 160 Abs. 1 BV. 
(oder eines Volksauftrags) verlangen, dass die Kantonsregierung ein Gesetz erarbeitet oder eine Massnahme ergreift. ${ }^{17}$ Die Anzahl gültiger Unterschriften sowie die Fristen sind je nach Kanton und befasstem Geschäft unterschiedlich. Auch die Häufigkeit, mit der das Initiativrecht erfolgreich genutzt wird, variiert erheblich. Wurden im Kanton Zürich beispielsweise von 1993 bis 2013 zwanzig von insgesamt 66 Initiativen angenommen, so haben die Stimmberechtigen des Kantons Uri im gleichen Zeitraum alle zehn Initiativen, die gültig zustande gekommen sind, abgelehnt. In derselben Zeitdauer waren im Kanton Waadt neun von neunzehn Initiativen an der Urne erfolgreich, im Kanton Jura eine von drei. Betrachtet man die Anzahl der Volksinitiativen, so schwingt der Kanton Zürich obenauf. Die Erfolgsquote liegt aber im Kanton Waadt mit fast 50 Prozent Gutheißung deutlich höher. Werden die dem Stimmvolk vorgelegten kantonalen Volksinitiativen mit der Anzahl Stimmbürgerinnen und Stimmbürger verglichen, so fällt auf, dass in Uri in einem Zeitraum von 20 Jahren eine Volksinitiative pro 2600 Stimmbürgerinnen und Stimmbürger zustande gekommen ist, während im Kanton Waadt in denselben Jahren einer von 22000 Stimmbürgern eine Volksinitiative lanciert hat.

Als direktdemokratische Sonderfälle gelten weiterhin die beiden Kantone Appenzell Innerrhoden und Glarus, die an der Landsgemeinde als höchster kantonaler Instanz festhalten. In der Landsgemeinde versammeln sich alle stimmberechtigten Bürgerinnen und Bürger und entscheiden durch Emporheben des Stimmrechtsausweises (Glarus) bzw. der Hand (Appenzell Innerrhoden) über Verfassungsänderungen, Gesetze und größere Ausgaben. Weil unter diesen Umständen keine geheime Abstimmung gewährleistet ist, hat die Schweiz bei der Ratifikation des UNO-Pakts zu den bürgerlichen und politischen Rechten einen Vorbehalt angebracht. ${ }^{18}$ Mit ihren offen durchgeführten Abstimmungen werfen Landsgemeinden Fragen auf, die sich in ähnlicher Weise auch bei Bürgerforen stellen. Im Jahre 1995 musste das Schweizerische Bundesgericht beispielsweise die Frage beantworten, ob Landsgemeinden die Stimm- und Wahlfreiheit verletzen. ${ }^{19}$ Es stellte dabei zunächst fest, dass offene Abstimmungen »unter psychologischen und sozialen Gesichtspunkten Beeinflussungen des

17 Bei einer Volksmotion stellt eine bestimmte Anzahl Stimmbürgerinnen und Stimmbürger einen Antrag an das Kantonsparlament, das den Antrag zu behandeln hat; vgl. Auer, Staatsrecht der Schweizerischen Kantone, (Fn. 14), 441.

18 Botschaft betreffend den Beitritt der Schweiz zu den beiden internationalen Menschenrechtspakten von 1966 und zu einer Änderung des Bundesrechtspflegegesetzes vom 30. Januar 1991, BBl 19911198.

19 BGE 121 I 138. 
Stimmbürgers bewirken «20 könnten und ein gewisser Konformitätsdruck nicht ausgeschlossen sei. Es wies auch darauf hin, dass ein Teil der Stimmbürgerschaft, namentlich betagte und kranke Personen, aus unverschuldeten Gründen an der Versammlungsdemokratie nicht teilnehmen könne, die Beteiligung generell tief sei und das Abstimmungsergebnis nicht genau ermittelt, sondern nur geschätzt werden könne (sog. Mehren). Diesen Nachteilen seien aber die Vorteile der Landsgemeinde gegenüberzustellen, namentlich die Bürgernähe, der direkte Kontakt zwischen Behörden und Stimmbürgern, die Möglichkeit, Informationen aus erster Hand zu erhalten und zu diskutieren und die Möglichkeit, »den Entscheidungsvorgang unmittelbar selbst mit[zu]erleben «21, diese Einschätzung gälte gleichermaBen für die Versammlungsdemokratien auf kommunaler Ebene. ${ }^{22}$

Sowohl im Kanton Appenzell Innerhoden als auch im Kanton Glarus kann an der Landsgemeinde jede und jeder Stimmberechtigte zu beliebigen Fragen das Wort ergreifen. Dass dies wirkungsvoll geschehen kann, illustrierte im Jahre 2006 eindrücklich eine Glarner Landsgemeinde: Landrat und Regierungsrat hatten vorgeschlagen, die Zahl der Gemeinden von derzeit 27 auf zehn zu reduzieren, um größere und finanziell stabilere Gemeinden zu schaffen. Der Vorstoß einer Einzelperson, das Kantonsgebiet in Zukunft nur noch auf drei Gemeinden aufzuteilen, wurde an der Landsgemeinde überraschend gutgeheißen. Die Beschwerde eines Stimmbürgers, der geltend gemacht hatte, die Stimmbürgerinnen und Stimmbürger seien durch den Vorschlag überrumpelt worden, wies das Verwaltungsgericht des Kantons ab. Auch das Bundesgericht schützte diesen Entscheid und verwies auf die deliberative Funktion der Abänderungsanträge:

»Das Recht, Abänderungsanträge zu stellen, hat zur Folge, dass die Stimmberechtigten, anders als bei einer Urnenabstimmung, eine Vorlage nicht nur annehmen oder verwerfen können, sondern gestaltend auf eine Vorlage einwirken können. Dies stellt gerade den Sinn der Versammlungsdemokratie und ihr >demokratischer Mehrwert gegenüber der Urnendemokratie dar. Die Stimmberechtigten haben mit Abänderungsanträgen an der Versammlung zu rechnen. «23

20 BGE 121 I 138 E. 4a.

21 BGE 121 I 138 E. 4a.

22 Ebd.

23 BGE 132 I 291 E. 4.1. 
Aufgrund einer Volksinitiative wurde im Jahr darauf eine außerordentliche Landsgemeinde einberufen, die jedoch den ursprünglichen Beschluss mit großem Mehr bestätigte.

Volksinitiativen spielen auch auf der Ebene der gegenwärtig 2255 Gemeinden eine wichtige Rolle. Während größere Gemeinden in der Regel ein Gemeindeparlament kennen, das mit der Gesetzgebung und weiteren Aufgaben betraut ist, gibt es zahlreiche kleinere Gemeinden, in denen die in der Gemeindeversammlung zusammenkommenden Stimmbürgerinnen und Stimmbürger den Gesetzgeber bilden und zahlreiche Aufgaben (nicht nur rechtsetzende) direktdemokratisch wahrnehmen. ${ }^{24}$ Die Gemeindeversammlung bietet dem Stimmvolk die Möglichkeit, seine aus dem kantonalen Recht und der Gemeindeordnung fließende Zuständigkeit direkt wahrzunehmen. Wie die Landsgemeinde erlaubt auch die Gemeindeversammlung die öffentliche Diskussion und ermöglicht einzelnen Stimmbürgerinnen und Stimmbürgern, Vorschläge abzuändern, zu ergänzen oder Gegenvorschläge zur Abstimmung zu bringen. Vor allem in kleineren Gemeinden - die durchschnittliche Einwohnerzahl der Schweizer Gemeinden liegt trotz zahlreichen Fusionen weiterhin deutlich unter 3000 Personen - erübrigen sich damit meist Bürgerforen, wie sie in anderen Staaten an Bedeutung gewinnen. Sie finden auf der untersten Ebene in der Form der Gemeindeversammlung ohnehin statt und sind mit weitreichenden Entscheidbefugnissen ausgestattet. Gibt es dennoch andere Formen der Beteiligung, zielen sie auf den Einbezug weiterer Personen (Jugendlicher, Ausländerinnen und Ausländer), auf die Vorbereitung umstrittener Entscheide mit besonders Betroffenen oder auf die Entwicklung langfristiger, aber nicht verbindlicher Leitbilder $\mathrm{ab} .^{25}$

\subsection{Direkt-demokratische Vetorechte: Die Rotlichtfunktion}

Referendumsrechte ermöglichen dem Volk, Entscheide seiner Vertreterinnen und Vertreter abzulehnen. Wie die Initiativrechte sind sie auf eidgenössischer, kantonaler und kommunaler Ebenen vorgesehen und erfolgen entweder automatisch, wenn bestimmte Entscheide gefällt werden (obliga-

24 Auer, Staatsrecht der Schweizerischen Kantone (Fn. 14), 143 f.

25 Vgl. Abschnitt 4.2. Die Mitwirkung der Betroffenen im Planungsverfahren: Das Akzeptanzgenerierungssystem und Abschnitt 4.3. Die Partizipation der Engagierten an offenen Gemeindeforen: Das Orientierungssystem. 
torisches Referendum), oder wenn eine bestimmte Zahl von Stimmberechtigten dies verlangt (fakultatives Referendum).

\subsubsection{Die Referenden auf Bundesebene}

Die Schweizerische Bundesverfassung sieht für sämtliche Änderungen der Bundesverfassung, den Beitritt der Schweiz zu einer Organisation für kollektive Sicherheit (z.B. der NATO) oder zu einer supranationalen Gemeinschaften (z.B. der EU) sowie für dringlich erklärte Bundesgesetze ohne Verfassungsgrundlage, die länger als ein Jahr gelten sollen, obligatorische Referenden vor; diese Entscheide kommen nur zustande, wenn sie an der Urne von der Mehrheit der Schweizer Stimmbevölkerung (Volk) und von der Mehrheit der Kantone (Stände) gutgeheissen wird (qualifiziertes Mehr). ${ }^{26}$ Ein obligatorisches Referendum mit einfacher Mehrheit (nur das Volk muss zustimmen) findet statt, wenn eine Volksinitiative auf die Totalrevision der Bundesverfassung vorliegt, wenn eine Volksinitiative deren Teilrevision in der Form der allgemeinen Anregung verlangt und die Bundesversammlung diese ablehnt sowie wenn sich National- und Ständerat nicht einig sind, ob eine Totalrevision durchzuführen sei.

Von großer praktischer Bedeutung ist das fakultative Referendum, das 1874 mit der ersten Totalrevision der Bundesverfassung eingeführt wurde. Nach verschiedenen Revisionen kann es heute gegen Bundesgesetze, bestimmte Bundesbeschlüsse und gegen völkerrechtliche Verträge ergriffen werden, die unbefristet und unkündbar sind, den Beitritt zu einer internationalen Organisation vorsehen, wichtige rechtsetzende Bestimmungen enthalten oder deren Umsetzung, den Erlass von Bundesgesetzen, erfordern. Ein fakultatives Referendum kommt zustande, wenn 50000 Stimmberechtigte oder acht Kantone dies innerhalb von 100 Tagen seit der amtlichen Veröffentlichung des Erlasses verlangen. ${ }^{27}$ Fakultative Referenden erlauben es der Bevölkerung damit, wichtige Entscheide der Bundesversammlung an die Urne zu bringen, wo sie vom Volk gutgeheißen oder abgelehnt werden.

Es ist vor allem das fakultative Referendum, das die Konkordanzdemokratie der Schweiz hervorgebracht hat. Das Vetorecht stellt nämlich sicher, dass die Interessen aller möglichen Akteure berücksichtigt werden und

26 Art. 140 BV. Das Ergebnis der Volksabstimmung in einem Kanton gilt als Standesstimme dieses Kantons; vgl. Art. 142 Abs. 3 BV.

27 Art. $141 \mathrm{BV}$. 
ist - entgegen dem ersten Anschein - gerade kein Instrument der Mehrheitsdemokratie. Ende des 19. Jahrhunderts hatten die sogenannten Referendumsstürme der katholisch-konservativen Minderheit die damals noch ausschließlich aus Liberalen bestehende Landesregierung lahmgelegt. Die Liberalen, die damals dank dem Majorzwahlsystem auch in der Bundesversammlung die Mehrheit stellten, entschlossen sich deshalb 1891, einen ersten, 1919 einen zweiten Konservativen in den Bundesrat zu wählen. Dieser Entscheid zur Machtteilung war nicht großherzig, sondern pragmatisch und beruhte auf der Einsicht, dass nur Vorlagen, die auf einem breiten Konsens beruhen, vor dem Volk Bestand haben. Als in Folge der Einführung der Proporzwahl die Sozialdemokraten in großer Zahl in das Parlament gelangten, wurden bald auch sie in die Landesregierung eingebunden; als das Gleiche mit der Bauern-, Gewerbe- und Bürgerpartei (später Schweizerische Volkspartei) geschah, entstand die sogenannte Zauberformel, die alle wählerstarken Parteien in die Landesregierung einbindet. $^{28}$

Schon Arend Lijphart hatte darauf hingewiesen, dass die Referendumsrechte der Schweiz nicht reine Instrumente der Mehrheitsdemokratie seien. ${ }^{29}$ Tatsächlich erlauben sie es größeren und auch kleineren Gruppen, sich gegen Gesetze oder andere Erlasse zur Wehr zu setzen, die ihren Interessen zuwiderlaufen. Das fakultative Referendum steht deshalb stark im Dienste der Minderheiten, jedenfalls der organisierten, und hat zur Folge, dass jeder Vorlage lange Verhandlungen vorausgehen, die auf Konsens und Kompromisse zielen und verhindern sollen, dass wichtige Akteure den Eindruck erlangen, eine Vorlage lasse ihre Anliegen außer Acht. Im Vorfeld von Erlassen finden sich denn auch entscheidende deliberative Elemente: Wichtige politische Akteure werden möglichst frühzeitig über Gesetzesvorhaben informiert, sie erhalten Gelegenheit zur Stellungnahme, ihren Bedenken und Wünschen wird zumindest dann Rechnung getragen, wenn sie glaubhaft mit einem Referendum drohen können und deshalb mit erheblichen Verzögerungen (und dem möglichen Scheitern der Vorlage an der Urne) zu rechnen ist. Als Mehrheitssystem zeigt sich das Verfahren allerdings gegenüber jenen Gruppen, die von diesen Verfahren ausgeschlossen sind (namentlich Ausländerinnen und Ausländer) oder die zu wenig organisiert, finanziert oder mediatisiert sind, um sich wirksam

28 Häfelin et al., Schweizerisches Bundesstaatsrecht (Fn. 4) 494; Tschannen, Staatsrecht der Schweizerischen Eidgenossenschaft (Fn. 10), 48 und 505 f.

29 Arend Lijphart, Patterns of Democracy (Yale University Press, 1999), 231. 
Gehör zu verschaffen (namentlich Straffällige, neu eingebürgerte Schweizerinnen und Schweizer, Fahrende, etc.).

Referenden betreffen in aller Regel rechtssetzende Vorlagen. Sie werden vereinzelt aber auch eingesetzt, um Einzelfallentscheide direktdemokratisch abzusichern. Um die Akzeptanz der Eisenbahn-Alpentransversale sicherzustellen, verabschiedete die Bundesversammlung beispielsweise das Bundesgesetz über den Bau der Eisenbahn-Alpentransversale (NEAT), das wie jedes Bundesgesetz - dem fakultativen Referendum unterstand. Dieses wurde auch ergriffen; das Stimmvolk hieß das Infrastrukturprojekt jedoch im Jahre 1992 bei einer Stimmbeteiligung von 45.9 Prozent mit 63.6 Prozent der Stimmen gut. ${ }^{30}$ Einige Jahre später wurde auch die Finanzierung direktdemokratisch legitimiert. 1998 wurde das Referendum gegen den Bundesbeschlusses über Bau und Finanzierung von Infrastrukturvorhaben des öffentlichen Verkehrs ergriffen; bei einer tieferen Stimmbeteiligung von 38.3 Prozent wurde der Beschluss von 63.5 Prozent der Stimmenden gutgeheiBen. ${ }^{31}$ Gegnerinnen und Gegner des Projekts hatten damit zweimal die Gelegenheit, dieses an der Urne in Frage zu stellen, was den Bau der NEAT ohne Zweifel verzögert hat, aber möglicherweise dafür verantwortlich war, dass sich das "Jahrhundertprojekt" verwirklichen ließ, ohne durch Protestveranstaltungen behindert zu werden.

\subsubsection{Die Referenden auf kantonaler und kommunaler Ebene}

Auch die Kantone sowie die Gemeinden mit Parlament kennen als Vetorecht das Referendum. Sie unterscheiden ebenfalls zwischen obligatorischen Referenden, die automatisch stattfinden, wenn bestimmte Beschlüsse gefällt werden, und fakultativen Referenden, die durch Unterschriftensammlungen oder andere Mechanismen ausgelöst werden müssen. In den Kantonen und Gemeinden findet sich dabei eine große Vielfalt an Volksrechten, die höchst unterschiedlich ausgestaltet sind.

Obligatorische Referenden finden aufgrund der bundesrechtlichen Homogenitätsklausel bei Verfassungsänderungen statt. ${ }^{32} \mathrm{Oft}$ wird bereits die Frage, ob eine Totalrevision der Verfassung in Angriff genommen werden

30 Bundeskanzlei BK, »Volksabstimmung vom 27.09.1992« (2018) www.bk.admin.c h/ch/d/pore/va/19920927/index.html (abgerufen am 4. Januar 2018).

31 Bundeskanzlei BK, »Volksabstimmung vom 29.11.1998« (2018) www.bk.admin.c h/ch/d/pore/va/19981129/index.html (abgerufen am 4. Januar 2018).

32 Verfassungsänderungen müssen bereits aus Art. 51 Abs. 1 BV dem Volk vorgelegt werden; vgl. Auer, Staatsrecht der Schweizerischen Kantone (Fn. 14), 415 f. 
soll, dem obligatorischen Referendum unterstellt. Ebenso existieren obligatorische Referenden zu bestimmten Gesetzen, zu Gebietsänderungen, zu Ausgaben ab einem gewissen Schwellenwert (obligatorisches Finanzreferendum), zur Sanierung der Kantonsfinanzen und zu vielem mehr. Dem fakultativen Referendum unterstehen je nach Kanton Gesetze, Verordnungen, Staatsverträge, Ausgaben ab einer bestimmten Höhe (fakultatives Finanzreferendum) oder Parlamentsbeschlüsse. ${ }^{33}$ Als bedingt fakultativ gilt ein Referendum, wenn eine Vorlage, für die das obligatorische Referendum vorgesehen ist, dem fakultativen unterstellt wird, wenn eine qualifizierte Mehrheit der Vertreterinnen und Vertreter des Parlaments der Vorlage zugestimmt hat. ${ }^{34}$ Verschiedene Kantone sehen diese Möglichkeit für bestimmte Gesetze, internationale oder interkantonale Verträge, Ausgabenbeschlüsse oder Pläne vor.

Ergreifen die Stimmbürgerinnen und Stimmbürger ein konstruktives Referendum, so legen sie gegen eine Vorlage ihr Veto ein, stellen dieser aber einen ausformulierten Gegenvorschlag gegenüber. ${ }^{35}$ Das konstruktive Referendum kombiniert damit Veto- und Vorschlagsrechte und erlaubt die direkte Beteiligung des Volks an der Gesetzgebung. Es verhindert, dass Vorlagen wegen einzelner Aspekte, die keine Zustimmung finden, zur Gänze zu Fall gebracht werden. Da es aber mit erheblichen praktischen Schwierigkeiten verbunden ist (namentlich wenn verschiedene Gruppen gleichzeitig ein konstruktives Referendum ergreifen), wurde es im Kanton Zürich 2012 nach sieben Jahren Experimentierphase allerdings wieder abgeschafft. ${ }^{36}$

\section{Schranken der direktdemokratischen Mitbestimmung}

Die Ausübung der Volkrechte ist an die Regeln gebunden, die die Verfassungen und Gesetze vorgeben. Im Folgenden sollen dabei die formellen

33 Die Anzahl geforderter Unterschriften von Stimmbürgern variiert je nach Kanton. Die Anforderungen in den französischsprechenden Kantonen sind deutlich höher als in den gemischt bzw. deutschsprechenden Kantonen. Die Frist, bis wann die Unterschriften gesammelt sein müssen, variiert zwischen 30 Tagen und 3 Monaten; vgl. Auer, Staatsrecht der Schweizerischen Kantone (Fn. 14), $417 \mathrm{ff}$.

34 Die geforderte qualifizierte Mehrheit ist in den Kantonen sehr unterschiedlich; vgl. ebd., $421 \mathrm{f}$.

35 Die Kantone Bern und Nidwalden kennen das konstruktive Referendum; vgl. ebd., 422.

36 M.w.H. Andreas Glaser, Uwe Serdült und Evren Somer, »Das konstruktive Referendum - ein Volksrecht vor dem Aus?«, (2016) 10 AJP, 1354. 
Vorschriften, die sich auf die Verfahren beziehen, weitgehend außer Acht gelassen und vor allem die materiellen Schranken erläutert werden, denen die direktdemokratische Mitbestimmung unterworfen ist. Die Frage nach den Vorlagen, über die das Volk aufgrund ihres Inhalts nicht entscheiden soll bzw. sollte, stellt sich nur bei Vorschlagsrechten, nicht bei Vetorechten, und ist für die kantonale und kommunale Ebene im Wesentlichen beantwortet: Volksinitiativen sind immer dann ungültig, wenn sie übergeordnetes Recht verletzen; darüber hinaus können die Kantonen und Gemeinde ihre Volksrechte weiteren inhaltlichen Schranken unterwerfen.

Die inhaltlichen Schranken, denen die eidgenössische Volksinitiative auf Teilrevision der Bundesverfassung untersteht, werden hingegen seit einiger Zeit kontrovers diskutiert. Im Folgenden soll kurz auf die Rolle der Bundesversammlung (3.1) und des Bundesgerichts (3.2) bei der Durchsetzung der materiellen Schranken demokratischer Sachentscheide hingewiesen und festgestellt werden, dass die Vernachlässigung rechtstaatlicher Prinzipien zu zahlreichen Schwierigkeiten und zu Gefahren für Einzelne und Minderheiten führt.

\subsection{In dubio pro populo zum Ersten: Zurückhaltende Prüfung durch die Bundesversammlung}

Ist eine eidgenössische Volksinitiative auf Teilrevision der Bundesverfassung zustande gekommen, so entscheidet die Bundesversammlung gestützt auf eine Vorprüfung durch den Bundesrat über deren Gültigkeit. Nur drei kodifizierte und ein ungeschriebener Grund erlauben es National- und Ständerat, ausformulierte Volksinitiativen für ungültig zu erklären: ${ }^{37}$

- Eine Verletzung der Einheit der Form (die Initiative wählt nicht klar zwischen der allgemeinen Anregung und dem ausgearbeiteten Entwurf, sondern enthält Bestandteile, die der Ausarbeitung des Parlaments bedürften, und solche, die unmittelbar Anwendung finden könnten);

- Eine Verletzung der Einheit der Materie (die Initiative wirft gleichzeitig verschiedene Fragen auf, die nicht in einem so engen sachlichen $\mathrm{Zu}$ sammenhang stehen, als dass die Stimmberechtigen sie ohne weiteres mit einem »ja« oder »nein« beantworten könnten);

37 Vgl. Häfelin et al., Schweizerisches Bundesstaatsrecht (Fn. 4), 533 ff.; Tschannen, Staatsrecht der Schweizerischen Eidgenossenschaft (Fn. 10), 556 ff. 
- Ein Verletzung der zwingenden Bestimmungen des Völkerrechts (die Initiative steht im Widerspruch zu völkerrechtlichen Verpflichtungen, denen sich die Schweiz nicht entziehen kann);

- Die Undurchführbarkeit der Volksinitiative, die allerdings eine objektive sein muss und sich nicht aus praktischen (auch schwerwiegenden) Schwierigkeiten der Durchführung ergeben darf.

Dass die Bundesversammlung und nicht das Bundesgericht dafür zuständig ist, über die Gültigkeit von Volksinitiativen zu entscheiden und dass ihr Entscheid endgültig ist, ${ }^{38}$ zeigt, dass der Verfassungsgeber die Frage nach den Schranken der Volksrechte nicht (nur) nach rechtlichen, sondern (auch) nach politischen Kriterien beantwortet haben will. In der Praxis fällt das Parlament seine Entscheide nach dem Grundsatz in dubio pro populo. Zum einen erklärt es auch Volksinitiativen für gültig, die mehrere Sachfragen miteinander kombinieren (z.B. die ECOPOP-Initiative, die gleichzeitig die Zuwanderung in die Schweiz begrenzen und weltweit staatliche Mittel zur Förderung der freiwilligen Familienplanung einsetzen wollte). Zum andern legt es die einzige materielle Schranke, die zwingenden Bestimmungen des Völkerrechts, eng aus. Nach dem Willen des Verfassungsgebers müsste der Ausdruck autonom ausgelegt, ${ }^{39}$ doch hat die Bundesversammlung den verfassungsrechtlichen Ausdruck »zwingende Bestimmungen des Völkerrechts« bis anhin mit dem völkerrechtlichen ius cogens gleichgesetzt und damit heteronomo und eng verstanden. ${ }^{40}$ Es qualifiziert deshalb unkündbare völkerrechtliche Verträge, wie etwa die beiden UN-Pakte, und im europäischen Raum zwingende Bestimmungen des Völkerrechts, wie etwa die EMRK, nicht als "zwingende Bestimmungen des Völkerrechts" und ermöglicht dadurch Volksabstimmungen über Vorlagen, die im Falle ihrer Gutheißung nicht umgesetzt werden können, ohne wesentliche völkerrechtliche Verpflichtungen der Schweiz zu verletzen. Bei verschiedenen Volksinitiativen, die Bundesrat und Bundesversammlung für gültig erklärt, aber zur Ablehnung empfohlen hatten (z.B. bei der Minarett- , der Ausschaffungs-, der Verwahrungs- und der Masseneinwanderungsinitiative) war zwar unbestritten, dass sie im Widerspruch

38 Art. 173 Abs. 1 lit. f sowie Art. 189 Abs. 4 BV; Epiney und Diezig, »Kommentar zu Art. $139 \mathrm{BV} \ll$ in Waldmann, Belser und Epiney (Hrsg.), Basler Kommentar (Fn. 5), N 20.

39 Botschaft über eine neue Bundesverfassung vom 20. November 1996, BBl 1996 448.

40 Häfelin et al., Schweizerisches Bundesstaatsrecht (Fn. 4), 534 f.; Tschannen, Staatsrecht der Schweizerischen Eidgenossenschaft (Fn. 10), 559 f. 
zu völkerrechtlichen Verpflichtungen der Schweiz standen. Da sie aber kein völkerrechtliches ius cogens verletzten, führten sie zu einem obligatorischen Referendum und - nach ihrer Annahme durch Volk und Stände - zu neuen Verfassungsbestimmungen, die nicht nur zu Widersprüchen innerhalb der Verfassung führten, sondern auch zu teilweise ungelösten Konflikten mit dem Völkerrecht.

Bereits die erste erfolgreiche Volksinitiative aus dem Jahre 1893, die ein Schächtverbot in die Bundesverfassung einfügte und nur am Rande tierschützerische Anliegen verfolgte, sondern vielmehr antisemitischem Gedankengut verpflichtet war, machte deutlich, dass Mehrheitsentscheide Gefahren für Minderheiten mit sich bringen können. ${ }^{41}$ Zwar verfolgen die weitaus meisten Initiativen wirtschaftliche, ökologische, sozial- oder verkehrspolitische Ziele, doch zeigte das im Jahre 2009 gutgeheißene Minarettverbot, dass die Gefahr der Sündenbock-Verfassungsgebung nicht gebannt ist. ${ }^{42}$ Die im Unterschriftenstadium gescheiterte Volksinitiative »Todesstrafe bei Mord mit sexuellem Missbrauch" warf die besorgte Frage auf, ob auch ein solcher Vorstoß, da nicht im Widerspruch zum völkerrechtlichen ius cogens, für gültig erklärt werden müsste. Das Gleiche galt für die als Witz gedachte Reaktion auf die (erfolgreiche) Ausschaffungsinitiative »Zur Ausschaffung krimineller Männer«.

Dass in den letzten Jahren verschiedene Volksinitiativen gutgeheißen worden sind, die zu Widersprüchen innerhalb der Verfassung und zu Unvereinbarkeiten mit den völkerrechtlichen Verpflichtungen der Schweiz geführt haben, hat der Frage nach einer Beschränkung des Initiativrechts neue Aktualität verliehen. ${ }^{43}$ Sollten Initiativen, die den Grundwerten der Verfassung, den Grund- und Menschenrechten oder deren Kerngehalten, dem Völkerrecht oder wenigstens fundamentalen oder unkündbaren Verträgen widersprechen, nicht für ungültig erklärt werden und dies möglichst früh (also vor der Unterschriftensammlung) und auf möglichst rechtsstaatliche Weise (also vom Bundesgericht)? Obwohl zahlreiche Vorschläge dieser Art diskutiert wurden, hat sich bislang keiner auch nur

41 Christian Bolliger, „Die eidgenössische Volksabstimmung über das Schächtverbot von 1893«, in Adrian Vatter (Hrsg.), Vom Schächt-zum Minarettverbot - Religiöse Minderheiten in der direkten Demokratie (Verlag Neue Zürcher Zeitung, 2011), $70 \mathrm{ff}$.

42 Vgl. Vatter (Hrsg.), Vom Schächt-zum Minarettverbot (Fn. 41).

43 Zum Ganzen Epiney und Diezig, »Kommentar zu Art. 139 BV «(Fn. 38), N 48 ff.; Häfelin et al., Schweizerisches Bundesstaatsrecht (Fn. 4), 537 f.; Tschannen, Staatsrecht der Schweizerischen Eidgenossenschaft (Fn. 10), $561 \mathrm{ff}$. 
soweit durchzusetzen vermocht, dass er weiterverfolgt worden wäre. ${ }^{44} \mathrm{Als}$ nach der Annahme der Ausschaffungsinitiative die Durchsetzungsinitiative lanciert wurde, um das Parlament, das sich mit der Umsetzung der neuen Ausschaffungsregeln schwertat, durch direkt anwendbare Verfassungsbestimmungen zu umgehen, kam zwar verbreitet das Gefühl auf, die Volksrechte befänden sich in einer Krise; von dieser scheinen sie sich jedoch so gut erholt zu haben, dass sämtliche Vorschläge zur Beschränkung der Volksrechte derzeit ruhen.

Dass die Bundesversammlung als politisches Organ vorwiegend nach politischen Kriterien entscheidet und davor zurückschreckt, Entscheide in Frage zu stellen, die bereits die Unterstützung von 100000 Personen (und die entsprechende Medienaufmerksamkeit) gefunden haben, zeigt sich auch, wenn das Parlament Änderungen von Kantonsverfassungen genehmigt, die im jeweiligen Kanton bereits die Zustimmung der Mehrheit der Bevölkerung gefunden haben. Nur in ganz seltenen Fällen kann es sich zu einer Nichtgenehmigung kantonaler Verfassungsnormen durchringen. ${ }^{45}$ So genehmigte es etwa das Gesichtsverhüllungsverbot, das im Kanton Tessin aufgrund einer Volksinitiative gutgeheißen worden war, trotz seinem fraglichen Verhältnis zur Religionsfreiheit und zum Diskriminierungsverbot. ${ }^{46}$

44 Vgl. Anforderungen an die Gültigkeit von Volksinitiativen - Prüfung des Reformbedarfs - Bericht der Staatspolitischen Kommission des Ständerates vom 20. August 2015, BBl 20157099 ff.; Bericht des Bundesrates zur Abschreibung der Motionen 11.3468 und 11.3751 der beiden Staatspolitischen Kommissionen über Massnahmen zur besseren Vereinbarkeit von Volksinitiativen mit den Grundrechten vom 19. Februar 2014, BBl 20132337.

45 Beispielsweise gewährleistete der Ständerat die Schwyzer Kantonsverfassung, obwohl das Bundesgericht das darin enthaltene Proporzwahlsystem des Kantons Schwyz als bundesrechtswidrig taxiert hat. Der Nationalrat versagte der Schwyzer Kantonsverfassung jedoch die Garantie. Der Ständerat garantierte sie wiederum und der Nationalrat lehnte sie nochmals ab. Somit wurde sie schlussendlich nur wegen der Uneinigkeit der beiden Räte nicht gewährleistet, obwohl sie ein Wahlsystem vorsah, das ein rechtskräftiges Bundesgerichtsurteil als mit dem übergeordneten Recht unvereinbar erklärt hatte; Urteil des BGer 1C_407/2011 vom 19. März 2012, E. 6; vgl. Auer, Staatsrecht der Schweizerischen Kantone (Fn. 14), $226 \mathrm{f}$. und 240.

46 Geschäft des Bundesrates, »Kantonsverfassungen Bern, Uri, Solothurn, BaselStadt, Basel-Landschaft, Appenzell Ausserrhoden, Appenzell Innerrhoden, Tessin, Waadt und Jura. Gewährleistung« (2015) www.parlament.ch/de/ratsbetrieb/suche -curia-vista/geschaeft?AffairId=20140084 (abgerufen am 5. Januar 2018). 


\subsection{In dubio pro populo zum Zweiten: Beschränkte Prüfung durch das} Bundesgericht

Dass das Spannungsverhältnis zwischen Demokratie- und Rechtsstaatsprinzip in der Schweiz eher zugunsten der Demokratie gelöst wird, zeigt sich auch bei der gerichtlichen Überprüfung von Volksentscheiden. Hat die Bundesversammlung eine Volksinitiative für gültig erklärt, so kann dieser Entscheid vom Bundesgericht nicht überprüft werden - auch dann nicht, wenn rechtliche Zweifel daran bestehen, ob die Initiative die Einheit der Form, der Materie oder die zwingenden Bestimmungen des Völkerrechts achtet. $^{47}$

Das Schweizerische Verfassungsrecht kennt aber noch eine andere wichtige Schranke der Verfassungsgerichtsbarkeit: Bundesgesetze und völkerrechtliche Verträge sind von den Gerichten nach Art. 190 BV auch dann anzuwenden, wenn sie im Widerspruch zur Verfassung stehen. Gegenüber Bundesgesetzen und völkerrechtlichen Verträgen, die von der Bundesversammlung auf ihre Verfassungsmäßigkeit geprüft und vom Volk ausdrücklich oder stillschweigend (durch Verzicht auf das fakultative Referendum) gutgeheißen worden sind, müssen deshalb vom Bundesgericht in jedem Fall angewendet werden. Auch hier herrscht der Grundsatz in dubio pro populo oder die Überzeugung, dass es am Parlament und Volk, nicht an Richterinnen und Richtern, ist, in letzter Instanz darüber zu entscheiden, ob Gesetz und Völkerrecht im Einklang mit der Verfassung stehen. ${ }^{48}$ Das Bundesgericht kann zwar die Verfassungswidrigkeit eines Gesetzes feststellen, muss dieses aber dennoch anwenden. ${ }^{49}$

Nur wenn ein (durch die Verfassung immunisiertes) Gesetz dem (durch die Verfassung gleichermaßen immunisierten) Völkerrecht widerspricht, kann ihm das Bundesgericht die Anwendung verweigern. Der Vorrang des Völkerrechts wird vom Bundesgericht allerdings dann nicht durchgesetzt, wenn sich der Gesetzgeber bei der Verabschiedung des Bundesgesetzes der Völkerrechtswidrigkeit bewusst war und diese in Kauf nahm (Schubert Praxis). ${ }^{50}$ Diese Ausnahme wird jedoch durch eine Gegenausnahme

47 Epiney und Diezig, »Kommentar zu Art. 139 BV« (Fn. 38), N 20; Häfelin et al., Schweizerisches Bundesstaatsrecht (Fn. 4), 548.

48 Zum Ganzen Astrid Epiney, »Kommentar zu Art. 190 BV«, in Waldmann, Belser und Epiney (Hrsg.), Basler Kommentar (Fn. 5), N 23 ff.; Häfelin et al., Schweizerisches Bundesstaatsrecht (Fn. 4), $654 \mathrm{f}$.

49 Epiney, »Kommentar zu Art. 190 BV«(Fn. 48), N 35.

50 Häfelin et al., Schweizerisches Bundesstaatsrecht (Fn. 4), 600; Tschannen, Staatsrecht der Schweizerischen Eidgenossenschaft (Fn. 10), 173. 
eingeschränkt (PKK Praxis): Das fundamentale Völkerrecht, namentlich die EMRK, geht den Bundesgesetzen in jedem Fall vor. ${ }^{51}$ Damit verfügt die Schweiz im Ergebnis über eine versteckte Verfassungsgerichtsbarkeit, die es dem Bundesgericht erlaubt, Bundesgesetzen die Anwendung zu versagen, wenn diese die EMRK verletzen (deren Garantien in weiten Teilen mit den Grundrechten der Bundesverfassung übereinstimmen). Das Bundesgericht hat damit eine Möglichkeit gefunden, Einzelne und Minderheiten vor der übermässigen Einschränkung ihrer Menschenrechte durch Bundesgesetze zu schützen und zu verhindern, dass die Demokratie zu einer Tyrannei der Mehrheit ausartet. Es kann aber weiterhin EMRKwidrige Verfassungsrevisionen nicht verhindern und nur die Garantien der EMRK, nicht aber der Bundesverfassung vor dem Zugriff der Mehrheit schützen. Auch wenn es ihm damit gelungen ist, einen besseren Ausgleich zwischen Demokratie und Rechtsstaatlichkeit zu finden, so ist doch nicht zu übersehen, dass das Outsourcing der Frage nach den Schranken der direktdemokratischen Mitbestimmung an das Völkerrecht dieses unter Druck setzt. Dass dies nicht ohne Risiken geschehen kann, zeigte die Selbstbestimmungs-Initiative (»Schweizer Recht statt fremde Richter»), die den Vorrang der Bundesverfassung gegenüber dem Völkerrecht verankern und vorsehen wollte, dass nur noch jene völkerrechtlichen Verträge für das Bundesgericht massgebend sein sollten, deren Genehmigungsbeschluss dem Referendum unterstanden hat (was bei der EMRK nicht der Fall war). 52

In den Kantonen müssen Volksinitiativen eine Reihe von Gültigkeitsvoraussetzungen erfüllen, um zur Abstimmung zu gelangen. Es sind dies die Einheit der Form und der Materie, die Beachtung des höherrangigen Rechtes, die Wahl der richtigen Normstufe, eine hinreichend klare Formulierung, die praktische Durchführbarkeit sowie die Achtung des Rechtsmissbrauchsverbots. ${ }^{53}$ Zusätzlich sehen einige Kantone vor, dass Initiantinnen und Initianten ihrem Begehren eine Begründung beilegen müssen. Andere legen ausdrücklich fest, dass rückwirkende oder andere

51 Häfelin et al., Schweizerisches Bundesstaatsrecht (Fn. 4), 601; Tschannen, Staatsrecht der Schweizerischen Eidgenossenschaft (Fn. 10), $173 \mathrm{f}$.

52 Die Initative, die der Verfassungsgerichtsbarkeit durch die Hintertür der EMK wohl eine Ende gesetzt hätte, wurde im Oktober 2018 von Volk und Ständen an der Urne deutlich verworfen; vgl. zum Ganzen Eva Maria Belser und Rekha Oleschak-Pillai, Engagement of Swiss Courts with International Law: Looking at the Swiss Federal Supreme Court and its Ways of Dealing with Conflicts between Domestic Law and International Human Rights Guarantees (Oxford University Press, i.E.).

53 Vgl. Auer, Staatsrecht der Schweizerischen Kantone (Fn. 14), 432 ff. 
mit den rechtsstaatlichen Grundsätzen unvereinbare Initiativen ungültig sind. ${ }^{54}$ Kantonale Volksinitiativen, die diese Voraussetzungen nicht erfüllen, können durch die kantonal zuständige Behörde für ungültig erklärt werden. Die Gültig- oder Ungültigkeitserklärung wird auf Beschwerde von kantonalen Gerichten und in letzter Instanz vom Bundesgericht überprüft. 55

Während also auf eidgenössischer Ebene Volksinitiativen gültig sind, die zu Widersprüchen mit dem Völkerrecht oder der Bundesverfassung führen, so ist dies auf kantonaler und auf kommunaler Ebene nicht der Fall. Im Jahre 2012 hatte z.B. der Kantonsrat von Zürich die kantonale Volksinitiative "Steuerbonus für dich « auf Empfehlung des Regierungsrats für ungültig erklärt, weil sie bundesverfassungswidrig sei. ${ }^{56}$ Die Initiantinnen und Initianten wollten einmalig bei vermögenden natürlichen und juristischen Personen eine Umverteilungssteuer erheben und damit den unteren und mittleren Einkommen eine direkte Steuererleichterung ermöglichen. Die Modifizierung des Steuertarifs für ein Jahr sei nicht mit der Eigentumsgarantie (Art.26 BV) und der Besteuerung nach der wirtschaftlichen Leistungsfähigkeit (Art. 127 Abs. 2 BV) vereinbar, entschied das Parlament. Die dagegen erhobene Beschwerde wies das Bundesgericht ab. Es sah auch mit Blick auf das Verhältnismäßigkeitsprinzip keine Möglichkeit, die Initiative teilweise als gültig zu erklären.

Auch die freiburgische Volksinitiative "Gegen die Eröffnung eines `Zentrums Islam und Gesellschaft und eine staatliche Imam-Ausbildung an der Universität Freiburg « illustriert, dass parlamentarische und gerichtliche Kontrollen einen Ausgleich zwischen direktdemokratischer Mitbestimmung und rechtsstaatlichem Grundrechtsschutz gewährleisten und notwendig sind, um das Nebeneinander von Volks- und Menschenrechten zu sichern. Stimmbürgerinnen und Stimmbürger des Kantons Freiburg hatten 2015 eine als allgemeine Anregung ausgestaltete Volksinitiative eingereicht, die sich gegen ein neues wissenschaftliches Zentrum der Universität richtete. Der Grosse Rat kam zum Schluss, dass die Initiative gegen das Diskriminierungsverbot verstoße und erklärte die Ungültigkeit. Dagegen erhoben die Initiantinnen und Initianten Stimmrechtsbeschwerde an

54 Der Kanton Graubünden hat dieses Prinzip in seiner Kantonsverfassung festgeschrieben; vgl. Auer, Staatsrecht der Schweizerischen Kantone (Fn. 14), 436.

55 Vgl. ebd., 439 f.; Häfelin et al., Schweizerisches Bundesstaatsrecht (Fn. 4), 423.

56 Steuerbonus für dich, (2013) https://www.wahlen.zh.ch/abstimmungen/initiative n/iniliste_detail_test.php?id=59 (abgerufen am 5. Januar 2018); Urteil des BGer 1C_586/2013 vom 7. Oktober 2014. 
das Bundesgericht, das die Ungültigkeitserklärung des Kantonsparlaments stützte und die Beschwerde abwies. ${ }^{57}$

Im Falle der Fremdspracheninitiative im Kanton Graubünden urteilten die Gerichte überraschend zugunsten eines direktdemokratischen Entscheids. Das Anliegen der Initianten bestand darin, das Bündner Stimmvolk entscheiden zu lassen, ob die deutschsprachigen Schülerinnen und Schüler des Kantons in der Primarschule nur noch Englisch, die italienisch- und rätoromanisch sprechenden Kinder dagegen nur noch Deutsch als Fremdsprache lernen sollten. Zwei Gutachter sowie die Bündner Regierung und der Grosse Rat erachteten die in der Form der allgemeinen Anregung formulierte Volksinitiative als unvereinbar mit der Bundessowie der Kantonsverfassung. Sie erblickten darin eine Diskriminierung aufgrund der Sprache für die italienisch- und rätoromanisch sprechenden Schülerinnen und Schüler. Der Grosse Rat erklärte die kantonale Volksinitiative 2015 deshalb für ungültig. Das kantonale Verwaltungsgericht hob diesen Entscheid jedoch auf. Es kam zum Schluss, dass auch im Fall einer Annahme der Initiative bis am Ende der obligatorischen Schulzeit (Primar- und Sekundarschule) die gleichwertige Ausbildung bezüglich Fremdsprachkompetenzen aller Schüler im Kanton Graubünden gewährleistet sei. Im Jahre 2017 wies das Bundesgericht die Beschwerde gegen diesen Entscheid ab. ${ }^{58}$

Auch nach der Volksabstimmung können Verletzungen des übergeordneten Rechts durch kantonales und kommunales Recht jederzeit vor Bundesgericht geltend machen. Gegen kantonale Erlasse ist sowohl die abstrakte als auch die konkrete Normenkontrolle zugelassen; ${ }^{59}$ eine Ausnahme gilt dabei nur für die Kantonsverfassungen, die durch die Gewährleistung durch die Bundesversammlung immunisiert und erst dann der konkreten Normenkontrolle unterstehen, wenn es zu Neuerungen im übergeordneten Recht gekommen ist. ${ }^{60}$

57 BGE 143 I 129.

58 Urteil des BGer 1C_267/2016 vom 3. Mai 2017.

59 Art. 82 lit. a und b, Art. 86 Abs. 1 lit. d sowie Art. 87 des Bundesgesetzes über das Bundesgericht vom 17. Juni 2005 (SR 173.110, BGG); Auer, Staatsrecht der Schweizerischen Kantone (Fn. 14), $615 \mathrm{ff}$.

60 So geschehen im Frauenstimmrechtsfall betreffend die Kantonsverfassung Appenzell Innerrhoden. Das Bundesgericht legte die kantonale Verfassungsbestimmung im Lichte des neuen Gleichstellungsartikel der Bundesverfassung aus und urteilte, dass der Begriff Landleute und Schweizer sowohl die Einwohner als auch die Einwohnerinnen des Kantons umfasst; BGE 116 Ia 359 E. 10. 
Einige Kantone kennen verschiedene Verfahren, die es Gerichten erlauben, Erlasse oder Volksinitiativen abstrakt auf ihre Übereinstimmung mit höherrangigem Recht zu prüfen. ${ }^{61}$ Je nach Kanton können Normen vor oder nach ihrem Inkrafttreten angefochten werden. Antragsberechtigt sind alle natürlichen und juristischen Personen des betreffenden Kantons, die zumindest ein virtuelles Interesse geltend machen können, sowie in einigen Kantonen die Behörden. Ausser drei kennen auch alle Kantone die konkrete Normenkontrolle, bei der vorfrageweise anhand einer Verfügung die Verfassungsmässigkeit einer Norm überprüft wird. ${ }^{62}$

Gegen Entscheide der Gemeindeversammlung wie auch des Gemeindeparlaments stehen Stimmbürgerinnen kantonale Rechtsmittel zur Verfügung. Nach Erschöpfung des Instanzenzugs können sie sich mit Stimmrechtsbeschwerde an das Bundesgericht wenden. ${ }^{63}$

\section{Formen der deliberativen Demokratie}

Die zahlreichen Formen direktdemokratischer Vorschlags- und Vetorechte führen dazu, dass Parlamente und Regierungen der Schweiz in einer besonders nahen Verbindung zum Volk stehen. Mit Hilfe von Initiativrechten können die Stimmberechtigten auf die Agenda ihrer Vertreterinnen und Vertreter einwirken, mit dem Mittel der Referendumsrechte deren Entscheide zu Fall bringen. Auch wenn Volksabstimmungen letztendlich in einen Ja-Nein-Entscheid münden, befördern sie im Vorfeld die Deliberation: Ein vielfältig zusammengesetzter Bundesrat schmiedet hinter verschlossenen Türen Vorlagen, die einem breiten Konsens entsprechen, im Vernehmlassungsverfahren wird getestet, ob wichtige Akteure, namentlich Verbände, schwerwiegende Bedenken erheben, im National- und Ständerat werden Debatten geführt und Differenzen bereinigt, um am Schluss einen Erlass zu verabschieden, der weder Kantone noch Parteien oder andere interessierte Kreise zum Unterschriftensammeln motiviert oder doch so breit abgestützt ist, dass er ein allfälliges Referendum überstehen kann (4.1). Andere auf Mitsprache ausgerichtete und nicht direkt auf einen Volksentscheid gerichtete Verfahren finden sich vor allem im Raumplanungsrecht (4.2). Daneben lassen sich jedoch auch in der Schweiz,

61 Zum Ganzen Auer, Staatsrecht der Schweizerischen Kantone (Fn. 14), 603 und $611 \mathrm{ff}$.

62 Ebd., 618.

63 Ebd., 173 ff. und 613 f.; als Beispiel BGE 99 Ia 402. 
namentlich auf kommunaler Ebene, zahlreiche Experimente mit neuen Formen deliberativer Demokratie beobachten (4.3).

\subsection{Die Mitsprache der Interessierten im Rahmen von Vernehmlassungsverfahren: Das Frühwarnsystem}

Die Bundesverfassung verpflichtet die Bundesbehörden dazu, die Kantone, die politischen Parteien und die interessierten Kreise bei der Vorbereitung wichtiger Erlasse und anderer Vorhaben von großer Tragweite zur Stellungnahme einzuladen. ${ }^{64}$ Das Vernehmlassungsverfahren, das als eine »formalisierte Ausübung des Petitionsrechts ${ }^{65}{ }^{6}$ gilt, findet statt, wenn sich der Erlass oder das Vorhaben noch in der Vorbereitungs- oder Entwurfsphase befindet; ${ }^{66}$ es bezweckt die Beteiligung betroffener und interessierter Körperschaften und Personen, der Verbände und Kommissionen an der Meinungsbildung und Entscheidfindung im Bund und soll Aufschluss geben über die sachliche Richtigkeit, die Vollzugstauglichkeit und die Akzeptanz eines Vorhabens. ${ }^{67}$ Es hat bei Verfassungsänderungen, Gesetzesvorlagen und wichtigen völkerrechtlichen Vorträgen, also bei allen Vorhaben, die einem obligatorischen oder fakultativen Referendum unterstehen, zwingend stattzufinden und dient dazu, Referenden zu verhindern bzw. »referendumsfeste« Vorlagen zu erarbeiten. Als referendumsfest gelten dabei Vorlagen, bei denen die Anliegen all jener Kreise berücksichtigt worden sind, die genügend stark und organisiert sind, um ein Vorhaben durch ein Referendum zu verzögern oder zu Fall zu bringen. Darüber hinaus unterliegen auch Verordnungen und andere Vorhaben der Vernehmlassungspflicht, wenn sie "von grosser politischer, finanzieller, wirtschaftlicher, ökologischer, sozialer oder kulturellen Tragweite sind «.6 ${ }^{68}$ Damit stellt das Vernehmlassungsverfahren auch in jenen Bereichen die Beteiligung

64 Art. 147 BV.

65 Giovanni Biaggini, »Kommentar zu Art. 33 BV«, in Giovanni Biaggini (Hrsg.), Kommentar BV (Orell Füssli, 2007), N 1.

66 Thomas Sägesser, »Kommentar zu Art. 3 des Bundesgesetz über das Vernehmlassungsverfahren vom 18. März 2005 (SR 172.061, VlG) «, in Thomas Sägesser (Hrsg.), Handkommentar Vernehmlassungsgesetz (Stämpfli Verlag, 2006), N 7 ff.

67 Art. 2 VIG; das Verfahrens legt denn auch "nicht [Wert] auf die Information an die Adressaten, sondern auf die Information von den Adressaten « Patricia M. Schiess Rütimann, »Kommentar zu Art. 147 BV«, in Waldmann, Belser und Epiney (Hrsg.), Basler Kommentar (Fn. 5), N 8.

68 Art. 3 Abs. 1 lit. d VIG. 
der Öffentlichkeit an Vorhaben sicher, die von direktdemokratischen Vorschlags- und Vetorechten ausgenommen sind. ${ }^{69}$

Das Vernehmlassungsverfahren verhindert, dass Vorhaben weiterverfolgt werden, die von weiten Teilen des Volks, der Kantone oder einflussreichen Verbänden abgelehnt werden. ${ }^{70}$ Seine Bedeutung ist eng mit den Referendumsrechten verbunden: indem es darauf zielt, Referenden vorzubeugen, führt es oft $\mathrm{zu}$ »Konsensgesetzgebung«, die von großen Würfen absieht und den Anliegen und Bedenken möglichst weiter Kreise Rechnung trägt. Das Vernehmlassungsverfahren stellt aber in vielerlei Hinsicht auch eine wichtige Ergänzung der Volksrechte dar, indem es erstens eine Mitwirkung gewährleistet, bevor nur noch ein Ja-Nein-Entscheid möglich ist, indem es zweitens beim Erlass von Verordnungen und anderer Vorhaben stattfinden kann oder muss, die nicht dem Referendum unterstehen, und indem es drittens die Beteiligung von Akteuren ermöglicht, die von den politischen Rechten ausgeschlossen sind. An einem Vernehmlassungsverfahren können sich nämlich urteilsfähige natürliche und juristische Personen sowie privat- und öffentlich-rechtliche Organisationen und Institutionen unabhängig von ihrer Staatsangehörigkeit und ihrem Sitz beteiligen. ${ }^{71}$ Auch können bestimmte Einzelpersonen und Unternehmen zur Stellungnahme eingeladen werden, wenn sie eine besondere Nähe zum Gegenstand haben oder über Fachwissen verfügen; ${ }^{72}$ sie haben jedoch keinen Anspruch darauf.

Die eingegangenen Stellungnahmen werden zur Kenntnis genommen, gewichtet, ausgewertet und die Ergebnisse in einem Bericht zusammengefasst. ${ }^{73}$ Die Vernehmlassungsunterlagen, die Stellungnahmen und der Ergebnisbericht, der sich meist artikelweise mit den eingegangenen Stellungnahmen auseinandersetzt, sind öffentlich zugänglich. ${ }^{74}$ Deren Auswertung liegt im Ermessen der Behörden, die den Erlass vorbereiten; ein Anspruch

69 Vernehmlassungsverfahren können auch in Fällen durchgeführt werden, wo das Gesetz dies nicht vorsieht (Art. 3 Abs. 2 VIG).

70 Vgl. Thomas Sägesser, »Kommentar zu Art. 2 VlG« in Sägesser (Hrsg.), Handkommentar Vernehmlassungsgesetz (Fn. 66), N 35 ff.; Schiess Rütimann, »Kommentar zu Art. 147 BV« (Fn. 67), N 8 ff.

71 Vgl. Art. 4 Abs. 1 VlG; Thomas Sägesser, »Kommentar zu Art. 4 VlG« in Sägesser (Hrsg.), Handkommentar Vernehmlassungsgesetz (Fn. 66), N 8 ff.; Schiess Rütimann, "Kommentar zu Art. 147 BV« (Fn. 67), N 9, die findet, dass diese weite Regelung im Widerspruch zu Art. 147 BV und den politischen Rechten steht.

72 Sägesser, »Kommentar zu Art. 4 VlG« (Fn. 71), N 73.

73 Art. 8 VIG sowie Thomas Sägesser, „Kommentar zu Art. 8 VlG« in Sägesser (Hrsg.), Handkommentar Vernehmlassungsgesetz (Fn. 66), N 4 ff.

74 Art. 9 VIG. 
auf Berücksichtigung besteht nicht. Auch wenn die grosse Bedeutung der Vernehmlassungsverfahren unbestritten ist, so wird immer wieder Kritik an der Auswahl der Adressaten des Verfahrens, den kurzen Fristen und der Gewichtung der Stellungnahmen laut. ${ }^{75}$ Der Bundesrat, das Departement oder andere Akteure, die das Verfahren durchgeführt haben, fällen nach der Vernehmlassung einen Entscheid über das weitere Vorgehen, der im Wesentlichen politischen Kriterien verpflichtet ist. Auch wenn dabei manches undurchsichtig bleiben mag, so gilt als Regel doch, dass Vorhaben, die dem Referendum unterstehen, angepasst oder aufgegeben werden, wenn die Vernehmlassungsantworten zahlreicher oder einflussreicher Akteure als ernsthafte Referendumsdrohungen erscheinen. Betroffene, deren Interessen nicht durch referendumsfähige Akteure vertreten werden, können dagegen kaum damit rechnen, dass ihrer Stellungnahme zur »sachlichen Richtigkeit « erhebliches Gewicht zukommt; nicht zuletzt aus diesem Grund kann das Vernehmlassungsverfahren nicht als Bürgerbeteiligungsverfahren gelten. ${ }^{76}$

Vernehmlassungsverfahren finden auch auf kantonaler und kommunaler Ebene statt sowie überall dort, wo Rechtssetzung stattfindet (z.B. an Universitäten). Sie stehen oft in einem engen Bezug zu den direktdemokratischen Mitwirkungsrechten, gehen aber in Bezug auf Adressatenkreis und betroffene Erlasse in der Regel oft weit über diese hinaus. Ob eine Vernehmlassung durchgeführt werden muss, hängt je nach Kanton von der Erlassform (z.B. zu allen Verfassungsänderungen und Gesetzen) oder der Wichtigkeit bzw. Tragweite der infrage stehenden Erlasse ab. Einzelne Kantone kennen ständige Vernehmlassungsteilnehmer, andere lassen neben der schriftlichen Form der Stellungnahme auch die elektronische, mündliche oder anderen Formen der Anhörung zu oder organisieren öffentliche Vernehmlassungen in Form von Debatten. ${ }^{77}$

75 Vgl. Schiess Rütimann, »Kommentar zu Art. 147 BV« (Fn. 67), N 3.

76 Ebd., N 10.

77 Zum Ganzen Stefan Neubert, „Kantonale Vernehmlassungsverfahren im Vergleich«, in Schweizerische Gesellschaft für Gesetzgebung (SGG) und Schweizerischen Evaluationsgesellschaft (SEVAL) (Hrsg.), LeGes (Bundeskanzlei, 2008), $271 \mathrm{ff}$. 


\subsection{Die Mitwirkung der Betroffenen im Planungsverfahren: Das Akzeptanzgenerierungssystem}

Informations- und Mitwirkungsrechte, die nicht im unmittelbaren Zusammenhang mit den Volksrechten oder dem Vernehmlassungsverfahren stehen, finden sich vor allem im Planungs- und Baurecht. Das eidgenössische Raumplanungsgesetz schreibt vor, dass die Behörden die Bevölkerung über die Ziele und den Ablauf der Planungen unterrichten und dafür sorgen müssen, dass diese »in geeigneter Weise « mitwirken kann. ${ }^{78}$ Die Mitwirkungsmöglichkeiten sollen die Qualität und die Akzeptanz der Planung verbessern, besonders Betroffenen die Gelegenheit zur frühzeitigen Stellungnahme geben und die Partizipationsformen erweitern. Da es sich bei Raumplänen nicht um Gesetze handelt, bleibt es den Stimmbürgerinnen und Stimmbürgern verwehrt, mit direktdemokratischen Instrumenten auf diese einzuwirken. ${ }^{79}$ Mit der Beteiligung der Bevölkerung am Planungsverfahren, nicht jedoch am Planungsentscheid, soll einerseits sichergestellt werden, dass eine umfassende Interessenabwägung stattfindet und die Planung verbessert wird. Andererseits sollen berechtigte Anliegen frühzeitig erkannt werden und in die Planung einfließen, nicht zuletzt um späteren Rechtsmittelverfahren oder anderen Verzögerungen vorzubeugen. Diese Zielsetzung verlangt es, dass die raumplanerischen Informations- und Mitwirkungsrechte allen natürlichen und juristischen Personen, die von der Planung betroffen sind, ohne Rücksicht auf ihre Staatszugehörigkeit zustehen.

Der Bundesgesetzgeber hat nur Mindestanforderungen festgehalten und die nähere Ausgestaltung den betroffenen eidgenössischen, kantonalen oder kommunalen Behörden überlassen. Die gebräuchlichsten Informationskanäle umfassen Veröffentlichungen in den Medien, die öffentliche Auflage von Entwürfen und Informationsveranstaltungen. Die planende Behörde hat mindestens die Pflicht, die Planungsentwürfe freizugeben sowie Einwände oder Vorschläge entgegenzunehmen und materiell dazu Stellung zu nehmen. Die Informations- und Mitwirkungsrechte der Bevölkerung sind verbindlich und gerichtlich durchsetzbar. ${ }^{80}$

Auch wenn viele Kantone und Gemeinden sich bemühen, frühzeitig und umfassend über Planungen und Projekte zu informieren und den

78 Art. 4 Bundesgesetz über die Raumplanung vom 22. Juni 1979 (SR 700, RPG).

79 Zum Ganzen Bernhard Waldmann und Peter Hänni, »Kommentar zu Art. 4 RPG«, in Bernhard Waldmann und Peter Hänni (Hrsg.), Handkommentar Raumplanungsgesetz (Stämpfli Verlag, 2006), N 1 ff.

80 Ebd., N 5. 
Bedenken und Wünschen der Betroffenen Rechnung zu tragen, so kommt es doch regelmäßig zu Vorwürfen, die Bevölkerung würde zu spät informiert und könne, wenn überhaupt, nur noch pro forma mitwirken, wenn die Entscheide in Tat und Wahrheit schon gefällt seien. Von solchen Vorwürfen war vor kurzem der kantonale Gestaltungsplan des Kantons Zürich betroffen, der zwei neue Großprojekte - der Hochschulpark in der Stadt Zürich sowie der Innovationspark in Dübendorf - entscheidend voranbrachte, ohne die Bevölkerung an diesen Entscheiden angemessen zu beteiligen. Solche Generationenprojekte bedürften schon deshalb früher und vermehrter Mitwirkung, um sie später nicht an Einsprachen scheitern zu lassen, so die Kritik. ${ }^{81}$

Innovative Formen der Mitwirkung entstanden im Rahmen der dritten Rhonekorrektur, wo ein ständiges, die Grenzen der politischen Körperschaften überschreitendes Partizipationsgremium geschaffen wurde, dem sogar gewisse Entscheidkompetenzen zukamen. ${ }^{82}$ Die im Jahre 2008 durchgeführte öffentliche Vernehmlassung hatte zahlreiche Anliegen, Vorschläge und Gutachten zu Tage gefördert, die sich vornehmlich den Folgen des Projekts für die Landwirtschaft widmeten. Das Projekt wurde in der Folge überarbeitet und schliesslich von den Regierungen der beiden betroffenen Kantone Wallis und Waadt im Jahre 2016 genehmigt. ${ }^{83}$ Das Projekt gilt als einzigartiges »Ergebnis eines partizipativen Vorgehens auf kantonaler Ebene (kantonale Dienststellen, Verbände) und regionaler Ebene (Gemeinden, lokale Interessensvertreter) $\ll^{84}$

Vergleichbare, wenn auch informellere Vorgehensweisen haben sich die Schweizerischen Bundesbahnen (SBB) zu Eigen gemacht. Als in der Stadt Zürich verschiedene Areale der SBB, die für den Bahnverkehr nicht mehr benötigt wurden, einer Umnutzung zugeführt werden sollten, entschieden sich die SBB dafür, zusammen mit der lokalen Bevölkerung ein städtebauliches Konzept zu erarbeiten. Innerhalb von acht Monaten entstand so in

81 Vgl. Stefan Hotz, »Mitsprache statt Einsprache« Neue Zürcher Zeitung (Zürich, 18. Oktober 2017) www.nzz.ch/meinung/mitsprache-statt-einsprache-ld.1321740 (abgerufen am 5. Januar 2018).

82 Annina Schneider Fellmann, Neue Partizipationsformen in der Gemeindedemokratie (DIKE, 2013), 13; vgl. Canton du Valais/Kanton Wallis, "Rhone.vs« (2017) www.vs.ch/de/web/pcr/rhone.vs (abgerufen am 5. Januar 2018).

83 Canton du Valais/Kanton Wallis, "Generelles Projekt (GP-RG) genehmigt durch den Staatsrat« (2016) www.vs.ch/de/web/pcr/inhalt (abgerufen am 5. Januar 2018).

84 Canton du Valais/Kanton Wallis, »Das generelle Rhoneprojekt (GP-R3)« (2008) www.vs.ch/web/pcr/gpr3-was-ist-das?inheritRedirect=true (abgerufen am 5. Januar 2018). 
einem partizipativen Prozess, der von einem Moderator geleitet und durch ein 16-köpfiges Fachgremium sowie ein 15-köpfiges Autorenkollektiv unterstützt wurde, ein umfassendes Entwicklungskonzept. Die SBB führten insgesamt fünf Workshops durch, in welchen die Anwohnerinnen und Anwohner ihre Ideen und Wünschen vorbringen konnten. Anschließend wurden an einem weiteren Workshop die Vorschläge vom Fachgremium in Entwürfe gegossen und in nachfolgenden Veranstaltungen weiterdiskutiert. Das Experiment verlief so erfolgreich, dass die SBB diese Methode in Zukunft für all frei werdenden Areale anwenden will. ${ }^{85}$

\subsection{Die Partizipation der Engagierten an offenen Gemeindeforen: Das Orientierungssystem}

Weil die Konkordanz- und Abstimmungsdemokratie der Schweiz Kompromisse erfordert, gehören ein konsensorientierter Austausch von Argumenten und ein Abrücken von Positionen, die nicht mehrheitsfähig sind, zum politischen Alltag der Schweiz. Die Verhandlungen finden dabei allerdings im Wesentlichen zwischen den Spitzen der Parteien und Verbände statt und beruhen selten auf einer breiten Beteiligung des Stimmvolks. Es bleibt deshalb Raum für Foren, an denen sich Bürgerinnen und Bürger über das Gemeinwesen austauschen und längerfristige Leitlinien für die Entwicklung ihres Gemeinwesens abstecken. Solche Bürgerforen sind in letzter Zeit auf kommunaler Ebene vereinzelt entstanden; sie stellen keinen Ersatz, sondern eine Ergänzung der halbdirekten Demokratie dar. Während die Bürgerinnen und Bürger auf Gemeindeebene weiterhin zahlreiche wichtige Entscheide direkt in der Gemeindesversammlung oder an der Urne fällen, haben sie neu auch die Gelegenheit, gestaltend auf die Pläne und Leitbilder lokaler Gemeinwesen einzuwirken und den Behörden Orientierungslinien für ihr zukünftiges Tun vorzugeben.

Im Jahre 2006 hatte die Landsgemeinde des Kantons Glarus überraschend entschieden, die 25 Ortsgemeinden, 18 Schulgemeinden und neun Tagwen zu drei Einheitsgemeinden zusammenzuschließen. ${ }^{86}$ Die drei neuen Gemeinden Glarus Nord, Glarus und Glarus Süd sehen in ihren Ge-

85 Vgl. Dorothee Vögeli, »Mit 200 Leuten ein Konzept für Zehntausende Quadratmeter Stadtgebiet erarbeiten? Die SBB findet: Das geht. « Neue Zürcher Zeitung (Zürich, 4. Dezember 2017) www.nzz.ch/zuerich/die-sbb-wollen-nur-noch-mit-de r-bevoelkerung-planen-ld.1334797 (abgerufen am 5. Januar 2018).

86 Gemeinde Glarus, »Glarus in Kürze«www.gemeinde.glarus.ch/xml_1/internet/de/ application/d166/f173.cfm (abgerufen am 5. Januar 2018). 
meindeordnungen von 2011 alle vor, dass die Bevölkerung bei Grundsatzfragen und Fragen, die eine bestimme Gruppe besonders betreffen, einbezogen werden. ${ }^{87}$ In der Gemeinde Glarus Süd fand bereits im November 2011 eine erste Open-Space-Veranstaltung statt, an der sich ca. 60 Gemeindemitglieder beteiligten. ${ }^{88}$ Die Veranstaltung war inhaltlich und formell offen, im Plenum aufgeworfene Fragen und Themen wurden anschließend in Arbeitsgruppen weiterdiskutiert. Die Teilnehmerinnen und Teilnehmer konnten sich an maximal drei solcher Arbeitsgruppen beteiligen und waren in ihrer Wahl frei. Am Schluss wurde ein Bericht verfasst, der die Vorgehensweise und Zielsetzung der Veranstaltung darstellte und die vierzehn behandelten Themen und Projekte erläuterte. Diese waren sehr vielfältig und reichten von der Ablehnung der Idee, eine neue Sporthalle zu bauen, über die verbesserte Sensibilisierung der Bevölkerung für den UNESCO-Welterbe-Status von Glarus Süd und die Verbesserung der Hausarztversorgung bis zu diversen Anliegen bezüglich Tourismus und Standortattraktivität. Die Teilnehmerinnen und Teilnehmer repräsentierten ein weites Spektrum der Bevölkerung der Gemeinde Glarus Süd; sie kamen insbesondere aus dem Gesundheitswesen, Industrie- und Gewerbeunternehmen, Politik und Tourismus. Gestützt auf den Schlussbericht nahm sich die Gemeindeverwaltung den eingebrachten Themen und Projekten an, prüfte deren Umsetzbarkeit und Finanzierung und verwirklichte einzelne Projekte. Insgesamt waren die Organisatoren mit dem Vorgehen und den Ergebnissen sehr zufrieden und strichen vor allem das große Interesse und Engagement der Freiwilligen hervor sowie deren Bereitschaft, auch an der Weiterentwicklung der diskutierten Projekte mitzuwirken. ${ }^{89}$

Als bekanntes Beispiel innovativer Partizipation auf kommunaler Ebene gilt auch die im Kanton Luzern gelegene Gemeinde Horw. Horw hatte im Jahre 2006 eine Zukunfts- und eine Ergebniskonferenz durchgeführt mit

87 Schneider Fellmann, Neue Partizipationsformen (Fn. 82), 58.

88 Ebd., 58 f.; zum Vergleich: an der am 18. November 2011 durchgeführten Gemeindeversammlung haben sich 343 Gemeindemitglieder eingefunden. Insgesamt zählt die Gemeinde Glarus Süd ca. 9700 Einwohnerinnen und Einwohner. Glarus Süd, »Zahlen und Fakten« (31. Dezember 2017) www.glarus-sued.ch/xml 1/internet/de/application/d1/f212.cfm (abgerufen am 31. Dezember 2017) und »Protokoll: Gemeindeversammlung Glarus Süd« (18. November 2011) www.glar us-sued.ch/documents/111118_p_GV_GLS.pdf (abgerufen am 5. Januar 2018).

89 Zum Ganzen Schneider Fellmann, Neue Partizipationsformen (Fn. 82), 58 f.; Hans Peter Spälti, »`Mit dabei in Glarus Süd«: Entwicklung und Vertiefung von Themen der Gemeinde Glarus Süd - Schlussbericht der Veranstaltung vom 26. November 2011 (Februar 2012) www.glarus-sued.ch/documents/Schlussbericht_Mit dabei.pdf (abgerufen am 5. Januar 2018). 
dem Ziel, ein breit abgestütztes Leitbild für die Gemeinde zu erarbeiten und partizipativ Pläne für die kommenden 15 bis 20 Jahre zu entwickeln. ${ }^{90}$ Die Zukunftskonferenz wurde an zwei aufeinanderfolgenden Tagen mit ca. 200 Teilnehmenden durchgeführt. Die Bewohnerinnen und Bewohner wurden durch Inserate in Lokalzeitungen und durch persönliche Kontaktaufnahme von Mitgliedern der Spurgruppe, die sich vorgängig mehrmals getroffen hatte und einen repräsentativen Querschnitt der Bevölkerung abbilden sollte, auf die Veranstaltung aufmerksam gemacht. An der Konferenz und den Debatten beteiligten sich neben der Stimmbevölkerung und Einwohnerratsmitgliedern (Gemeindeparlament) auch Jugendliche und Einwohnerinnen und Einwohner mit ausländischer Staatsangehörigkeit. Zuerst diskutierten die Teilnehmer in Gruppen von acht bis zehn Personen, die sich immer wieder anders zusammensetzten, verschiedene Fragen rund um die Entwicklung von Horw sowie aktuelle Stärken und Schwächen der Gemeinde. Anschließend wurden Thesen, die der Gemeinderat vorbereitet hatte, in die Gespräche eingeführt; die Anwesenden konnten diese diskutieren und gegebenenfalls abändern bzw. neue Aspekte einbringen. Schließlich gewichteten die anwesenden Einwohnerinnen und Einwohner anhand der Vision Horw 2020 die einzelnen Projekte nach ihrer Wichtigkeit und Dringlichkeit. Es kristallisierten sich vor allem Projekte zur Steigerung der Lebensqualität (Halbinsel, bezahlbares Wohnen etc.), zur Innovationsförderung und für die Jugend als wichtig heraus. Gestützt auf diese Rückmeldungen und konkreten Vorschläge erarbeitete der Gemeinderat eine neue Version des Leitbilds. Spontan setzte dieser an der Zukunftskonferenz eine eintägige Ergebniskonferenz an. Dieser Einladung folgten über 100 Einwohnerinnen und Einwohner. An dieser Veranstaltung stellte jede Gemeinderätin und jeder Gemeinderat die Projekte vor, die sie oder er im eigenen Zuständigkeitsbereich zur Konkretisierung des neuen Leitbildes anstrebte. Die Einwohnerinnen und Einwohner waren abermals aufgerufen, kritisch Stellung zu nehmen und Änderungen bzw. zusätzliche Anliegen anzubringen. Das neue Leitbild wurde im Juni 2006 veröffentlicht und im September 2015 aktualisiert.

90 Gemeinde Horw, »Engagierte Zukunftsgestaltung: Zukunftskonferenz setzt Schwerpunkte für nachhaltige und qualitative Entwicklung (Blickpunkt, 24. Februar 2006) www.horw.ch/dl.php/de/20060223111045/01-12.pdf (abgerufen am 5. Januar 2018); Nico van der Heiden und Paul Krummenacher, Bringing the Backstage to the Front: The Role of Citizen Forums in Local Development Planning in Switzerland (University of Zurich, 2009), $8 \mathrm{f}$. 


\section{Schlussbemerkungen}

Die halbdirekte Demokratie der Schweiz erfreut sich großer Beliebtheit. Gegenwärtig sind alleine auf Bundesebene 26 Volksinitiativen in Vorbereitung und für vierzehn fakultative Referenden betreffend Änderungen von Bundesgesetzen oder -beschlüssen werden Unterschriften gesammelt. ${ }^{91}$ Auch auf kantonaler und kommunaler Ebene werden jedes Jahr unzählige Vorhaben vorgeschlagen und neue Ideen getestet, die Arbeit der Behörden kritisch begleitet und durch Referenden herausgefordert sowie - etwa im Rahmen von Finanzreferenden - für einen sorgsamen Umgang mit staatlichen Mitteln gesorgt. Wer unzufrieden ist, hat zahlreiche Möglichkeiten, sich Gehör zu verschaffen. Dass »die da oben« über die Köpfe des Volks hinweg entscheiden, ist ein Gefühl, das in der Schweiz wenig verbreitet ist. Die zahlreichen Beteiligungsmöglichkeiten beugen der Politikverdrossenheit und der Entfremdung zwischen Regierungen und Regierten vor. Direkte und deliberative Demokratie ist in der Schweiz denn auch nicht als Gegensatz zu verstehen. Auch wenn an der Urne keine Debatten stattfinden, sondern verbindliche Ja-Nein-Entscheide gefällt werden, so schaffen die zahlreichen Mitwirkungsmöglichkeiten doch einen Kontext, der dem Argumentieren, Diskutieren und Deliberieren förderlich ist. Weil aufgrund der Vorschlags- und Vetorechte ein ständiger Verhandlungszwang entsteht, gilt die Schweiz manchen als »deliberative Insel« in einem vorwiegend repräsentativ geprägten Umfeld. ${ }^{92}$ Die Abstimmungsdemokratie führt fast zwangsläufig zu einer Konkordanzdemokratie, in der nur Parteien und Politiker langfristig Erfolg haben, die für Argumente der Gegenseite offen und in der Lage sind, Kompromisse einzugehen.

Die Gefahr, dass Volksabstimmungen zu Polarisierungen führen können, ist dabei nicht von der Hand zu weisen. Pauschalisierung und Emotionalisierung erleichtern die Mobilisierung der Bevölkerung an der Urne. Hoch mediatisierte Parlamente sind vor diesen Gefahren aber ebenfalls nicht gefeit. Vieles weist außerdem darauf hin, dass der Kampagnencharakter von Volksabstimmungen weniger stark ausgeprägt ist, wenn diese regelmäßig und zu den unterschiedlichsten Sachfragen stattfinden. Der Polarisierung beugt auch vor, dass Referenden immer dann (aber nur dann)

91 Bundeskanzlei BK, »Vorlagen mit laufender Referendumsfrist (2018) www.admi n.ch/ch/d/pore/rf/ref_1_3_2_1.html (abgerufen am 5. Januar 2018).

92 Vgl. André Bächtiger, »Warum die Schweiz mehr Demokratie gut brauchen könnte: ein Plädoyer « (Tagung >Demokratie in der Krise - Stiftung Lucerna und NCCR Democracy, 15./16. März 2013) www.andre-baechtiger.ch/tl_files/baechtig er/pdf/DiskussionsbeitragDeliberation.pdf (abgerufen am 5. Januar 2018). 
stattfinden, wenn Verfassung und Gesetz dies vorsehen und nicht etwa, wenn es einer Behörde als opportun erscheint, das Volk zu befragen, um populär-populistischen Druck gegen andere Behörden oder gegen außen aufzubauen. In dieser Hinsicht unterscheiden sich Volksabstimmungen in der Schweiz denn auch fundamental von jenen, die etwa in Katalonien, Schottland, Großbritannien oder in Ungarn stattgefunden haben. Gerade die Vielzahl der Referenden und Referendumsdrohungen führt schließlich auch dazu, dass Gegnerinnen und Gegner nicht allzu sehr verunglimpft werden; kein politischer Akteur wird nämlich leichtfertig vergessen, dass eine bestimmte Partei oder Bevölkerungsgruppe bei einer anderen Sachfrage durchaus als Partner in Frage kommen kann.

Da sich die Schweiz aufgrund ihrer vielfältigen Initiativ- und Referendumsrechte als Hort der direkt-demokratischen Mitbestimmung sieht, nahm sie vor einigen Jahren mit Erstaunen zur Kenntnis, dass eine Studie der Universität Zürich und des Wissenschaftszentrums Berlin dem Land für seine Demokratie nur das Prädikat »mittelmässig« verlieh. Im sogenannten Demokratiebarometer, der 29 etablierte Demokratien verglich, belegte die Schweiz den vierzehnten Rang. ${ }^{93} \mathrm{Zu}$ den Indikatoren, bei denen die Schweiz schlecht abschnitt, gehörten die mangelnde Transparenz bei der Finanzierung der Schweizerischen Demokratie, die im Vergleich zu anderen Staaten beschränkte Rolle der Justiz bei der Kontrolle von Mehrheitsentscheiden sowie die geringe Stimmbeteiligung.

Das Ranking und die verwendeten Indikatoren wurden allerdings mit guten Gründen kritisiert. Die Stimmbeteiligung von Bevölkerungen, die alle vier Jahre die Gelegenheit haben, an Wahlen teilzunehmen, lässt sich nicht ohne Verfälschung mit jener von Bevölkerungen vergleichen, die fortwährend die Qualität der Arbeit ihrer politischen Repräsentantinnen und Repräsentanten überwachen, mehrmals jährlich Sachentscheide auf allen Stufen des föderalistischen Staats fällen und jederzeit die Möglichkeit haben, durch Initiativen Themen auf die politische Agenda zu setzen oder Vorlagen zu Fall zu bringen. ${ }^{94}$ Dennoch stellt sich die Frage,

93 NCCR Democracy, "Schweizer Demokratie im internationalen Vergleich mittelmässig« (Zürich, 27. Januar 2011) www.nccr-democracy.uzh.ch/news_events/ima ge/DemokratiebarometerCH_D.pdf/view (abgerufen am 5. Januar 2018); zur Erläuterung des angewandten Konzepts vgl. Marc Bühlmann et al., Demokratiebarometer: ein neues Instrument zur Messung von Demokratiequalität (Springer, 2012), $115 \mathrm{ff}$.

94 Vgl. zur Kritik an der Methode und der Verkennung der schweizerischen Eigenheiten etwa Sebastian Jäckle, Uwe Wagschal und Rafael Bauschke, Das Demokratiebarometer: »Basically Theory Driven«? (Springer, 2012), $99 \mathrm{ff}$. 
ob die traditionellen Einfluss- und Gestaltungsmöglichkeiten der Schweizer Demokratie den Ansprüchen an eine möglichst hohe demokratische Legitimation staatlicher Entscheide weiterhin genügen und ob sie nicht rechtsstaatliche Prinzipien, wie die Gewaltenteilung oder den Schutz der Einzelnen, vernachlässigen. Dies scheint jedoch weniger die Frage nach der Qualität der Demokratie zu betreffen, sondern vielmehr jene nach deren rechtsstaatlichen Schranken. Auch die dringend gebotenen Debatten über die Beschränkung der Volksrechte können jedoch relativiert werden: Die Volksrechte auf kantonaler und kommunaler Ebene werfen kaum rechtstaatliche Fragen auf, die sich nicht durch das übergeordnete Recht überzeugend beantworten ließen. Einzig die eidgenössische Volksinitiative auf Teilrevision der Bundesverfassung bedarf zusätzlicher Schranken, die verhindern, dass neue Verfassungsbestimmungen zur Abstimmung gelangen, die die Rechte Einzelner auf unzulässige Weise einschränken. Trotz verschiedener problematischer Volksinitiativen, die an die Hand genommen oder sogar gutgeheißen worden sind, hat es die Schweiz bis anhin versäumt, die Frage nach der direktdemokratischen Revision der Bundesverfassung überzeugend zu beantworten. Die gegenwärtige Auslagerung der rechtsstaatlichen Schranken an das Völkerrecht, namentlich die EMRK, setzt diese unter übermäßigen Druck, wovon die Selbstbestimmungsinitiative zeugte; sie verschleiert, dass das Volk nicht wegen der Strassburger Instanzen nicht alles darf, sondern weil Einzelne und Minderheiten vor der Tyrannei der Mehrheit geschützt werden müssen.

Besonders problematisch sind Volksentscheide, die Personen benachteiligen, die keine Möglichkeit haben, auf den Entscheid einzuwirken. Dies gilt neben allen Gruppen, die sich schlecht organisieren lassen, die Vorurteilen ausgesetzt oder finanzschwach sind, vor allem für alle Personen ohne Schweizer Staatsbürgerschaft. Weil die ausländische Wohnbevölkerung von der politischen Mitwirkung weitgehend ausgeschlossen ist, wird die Schweiz auch als Dreivierteldemokratie bezeichnet. ${ }^{95}$ Nur die Kantone Jura und Neuchâtel erkennen das Stimm- und aktive Wahlrecht der ausländischen Bevölkerung auf kantonaler Ebene an, die Kantone Genf, Fribourg, Jura, Neuchâtel und Waadt lassen die ausländische Bevölkerung auf kommunaler Ebene zu Wahlen zu und die Kantone Appenzell Ausserrhoden, Basel Stadt und Glarus erlauben ihren Gemeinden, dies vorzusehen. Dass sich über ein Viertel der ständigen Wohnbevölkerung nicht an politischen Entscheiden beteiligen kann, beeinträchtigt deren Legitimität.

95 Zum Ganzen Wolf Linder, Schweizerische Demokratie - Institutionen, Prozesse, Perspektiven (Haupt Verlag, 2005), 60. 
Auch der Grundsatz in dubio pro populo verliert in dem Maße an Überzeugungskraft, als Menschen die Folgen von Entscheiden tragen, die nicht zum (Stimm-)Volk gehören. Rechtsstaatliche Kontrollen (etwa betreffend der Verhältnismässigkeit von Maßnahmen gegenüber Ausländerinnen und Ausländer) erscheinen deshalb als unerlässlich.

Auch andere Entwicklungen bringen Herausforderungen für die direktdemokratische Mitbestimmung mit sich. Zahlreiche Gemeindefusionen und gesichtslose Agglomerationsgemeinden führen dazu, dass die Identifikation mit lokalen Gemeinwesen und das Interesse, an deren Gestaltung mitzuwirken, abnimmt. Die intensive horizontale Zusammenarbeit zwischen Gemeinden und Kantonen verschiebt die Macht von der Legislative auf die Exekutive und schwächt die Rolle der Parlamente und - erst recht - des Volks. Das Prinzip des Vorrangs höherrangigen Rechts erschwert es, demokratische Impulse von unten aufzunehmen und die häufige Rede von der Alternativlosigkeit staatlicher Vorhaben steht der Idee einer gestaltenden Mitwirkung des Volks entgegen. Die hohe Komplexität und der große Zeitdruck, die mit vielen Sachfragen vermeintlich oder tatsächlich verbunden sind, werfen ebenfalls Fragen nach der zukünftigen Ausgestaltung der direktdemokratischen Demokratie auf.

$\mathrm{Zu}$ den üblichen Rezepten zur Stärkung der Demokratie gehört die Verbesserung der politischen Bildung, insbesondere der stärkere Einbezug Jugendlicher. Wie die eidgenössischen Jugendsessionen verfolgen auch die über 50 Jugendparlamente der Schweiz, die sehr unterschiedlich organisiert sind, alle das Ziel, Jugendlichen die Gelegenheit zu geben, sich für die Anliegen junger Menschen einzusetzen, als Ansprechpartner für Behörden in Jugendfragen zu dienen und die politische Bildung zu stärken. ${ }^{96}$ Andere Akteure setzen auf die Digitalisierung, um ein breiteres Interesse an der politischen Mitbestimmung zu wecken und die Stimmbeteiligung zu erhöhen. Es geht dabei nicht nur um die elektronische Stimmabgabe, mit der gegenwärtig verschiedene Kantone erste Erfahrungen sammeln, ${ }^{97}$ sondern auch um die Idee, elektronisch den Graben zwischen Wählenden

96 Weitere Informationen unter www.jugendsession.ch und www.jugendparlament e.ch; als Beispiel Urs Bühler, »Im Trainingscamp der Demokratie« Neue Zürcher Zeitung (Zürich, 29. Oktober 2017) www.nzz.ch/zuerich/restaurantkritiken/im-tra iningscamp-der-demokratie-ld.1324813 (abgerufen am 5. Januar 2018).

97 Vgl. ch.ch, »Der elektronische Stimmkanal «www.ch.ch/de/demokratie/der-elektr onische-stimmkanal (abgerufen am 5. Januar 2018); Costa Vayenas, Democracy in the Digital Age (Arena Books, 2017), $2 \mathrm{f}$. 
und Gewählten zu überbrücken. Das Manifest für Digitale Demokratie ${ }^{98}$ fordert etwa, dass Bürgerinnen und Bürger via eine App die parlamentarischen Sessionen direkt mitverfolgen können. Die Idee ist dabei, dass Interessierte und Betroffene den Diskussionen nicht nur zuhören, sondern sich auch in die Debatten einschalten können. Im Dezember 2017 reichte Ständerat Damian Müller ein Postulat ein, mit dem der Bundesrat beauftragt wird, sich mit der Frage zu befassen, "wie mit den Möglichkeiten von 'Civic Tech Bürgerinnen und Bürger besser am politischen Prozess in der Schweiz teilnehmen können «.99

Bei diesen Forderungen, die auf eine Belebung der bestehenden Mitwirkungsprozesse zielen, muss bedacht werden, dass grenzenlose Transparenz und unmittelbare Reaktionen in der Form von likes der Deliberation sowohl förderlich als auch abträglich sein können. Daniel Kahnemans Bestseller "Schnelles Denken, langsames Denken« hat ins breite Bewusstsein gerückt, dass Entscheide, die unter Zeitdruck gefällt werden, sehr viel fehleranfälliger sind und öfter auf Vorurteilen und Herdentrieben beruhen, als Entscheide, die in aller Ruhe erwogen wurden. Das Vorbringen von Argumenten, die Auseinandersetzung mit Gegenargumenten, das vorurteilslose Abwägen, das Überwinden von Stereotypen, das Sich-überzeugen-lassen finden am besten in der Stille statt, denn sie brauchen nicht nur Zeit, sondern auch die Sicherheit, Fehler korrigieren und lernen zu können. All dies wird nicht gefördert, wenn Politikerinnen und Politiker andauernd im Scheinwerferlicht einer politisch aktiven (aber häufig nur Einzelinteressen verfolgenden) Öffentlichkeit stehen.

Neue Experimente mit deliberativer Demokratie, die für die Schaffung neuer oder die Verbesserung bestehender Foren des Austausches stehen, sind denn auch für die Schweiz von großem Interesse. Sie zielen darauf, neue oder bessere Möglichkeiten für Debatten zwischen Bürgerinnen und Bürgern und ihren Vertreterinnen und Vertretern zu schaffen und der Bevölkerung zu erlauben, Anliegen und Empfehlungen zu formulieren sowie Pläne und Visionen zu entwickeln. Wegleitend hat dabei zu sein, einen Austausch von Ideen zu ermöglichen, der möglichst ohne Zeit-, Profilierungs- und Anpassungsdruck erfolgt. Dies setzt in der Regel voraus, dass an den Foren keine Entscheide gefällt, sondern Bedürfnisse geklärt und Prioritäten gesetzt werden. Gerade die Tatsache, dass deliberative

98 Vgl. UZH Digital Society Initiative, »Manifest für digitale Demokratie« http://digi tal-manifest.ch/ (abgerufen am 5. Januar 2018).

99 Damian Meier, »Postulat 17.4017 - Die Chancen von `Civic Tech nutzen« (4. Dezember 2017) www.parlament.ch/de/ratsbetrieb/suche-curia-vista/geschaeft?AffairI $\mathrm{d}=20174017$ (abgerufen am 5. Januar 2018). 
Foren die bestehenden Behörden und Volksrechte ergänzen und nicht ersetzen, erlaubt es, deren Beschränkungen zu überwinden: Bürgerforen müssen sich nicht an die Grenzen der Gemeinwesen halten und können jene Personen beteiligen, die speziell interessiert oder von Entscheiden besonders betroffen sind. Sie können namentlich auch jugendliche Personen, die ausländische Bevölkerung, aber auch Vereine, Unternehmen und andere Akteure miteinbeziehen. Je breiter und offener diese Foren aufgestellt sind, umso eher wird der Turnverein-Demokratie vorgebeugt, bei der nur eine kleine engagierte Minderheit über eine unbeteiligte Mehrheit entscheidet. ${ }^{100}$ Überlegenswert wäre auch, die Mitglieder dieser Foren zumindest teilweise durch Los zu besetzen, um Personen an der Mitbestimmung zu beteiligen, die sich - aus welchen Gründen auch immer nicht für Wahlen aufstellen lassen und sicherzustellen, dass alle Bevölkerungsgruppen informiert sowie konsultiert werden und an der politischen Meinungsbildung mitwirken. ${ }^{101}$ So ausgestaltet könnten neue Bürgerforen und andere Formen der Beteiligung einer breiten Bevölkerung zweifellos einen wesentlichen Beitrag zur Erneuerung, Erweiterung und Vertiefung der halbdirekten Demokratien der Schweiz leisten.

100 van der Heiden und Krummenacher, Bringing the Backstage to the Front (Fn. 90), 2 ff.; Daniel Kübler et al., »Teil 1: Demokratieprobleme in Schweizer Gemeinden/Vertiefung der Demokratie? Warum Schweizer Gemeinden neue Formen der Bürgerbeteiligung einführen« in Daniel Kübler und Oliver Dlabac, Demokratie in der Gemeinde - Herausforderungen und mögliche Reformen (Schulthess, 2015), 93, $103 \mathrm{f}$. und $108 \mathrm{f}$.

101 Vgl. David von Reybrouck, Gegen Wablen - warum Abstimmen nicht demokratisch ist (Wallstein, 2016), mit zahlreichen Hinweisen auf historische und aktuelle Experimente mit Losverfahren; Gjon David und René Schuhmacher, »Ringen um Konsens kann gar nicht stattfinden - Streitgespräch zwischen Eva Maria Belser und Andreas Glaser«, (2017) 6 plädoyer, $6 \mathrm{ff}$. 